1 Hacettepe Journal of Mathematics and Statistics

Volume 46 (3) (2017), $449-468$

\title{
Optimal capital allocation with copulas
}

\author{
Zou Wei* and Xie Jie-hua ${ }^{\dagger \ddagger}$
}

\begin{abstract}
In this paper, we investigate optimal capital allocation problems for a portfolio consisting of different lines of risks linked by a Farlie-GumbelMorgenstern copula, modelling the dependence between them. Based on the Tail Mean-Variance principle, we examine the bivariate case and then the multivariate case. Explicit formulae for optimal capital allocations are obtained for exponential loss distributions. Finally, the results are illustrated by various numerical examples.
\end{abstract}

Keywords: Optimal Capital allocation; Tail Mean-Variance; Copula

2000 AMS Classification: 91B30; 60E05

Received : 07.03.2016 Accepted : 27.07.2016 Doi : 10.15672/ HJMS.20174620776

\section{Introduction}

A fundamental question in the fields such as quantitative risk management, insurance and finance is how much money to ask from a policyholder in a heterogeneous portfolio? A closely related question is how to allocate a given amount of capital between the different classes in a portfolio. The first question is known as the premium calculation problem and the second is the capital allocation problem. The two problems are well-known and have been studied, see e.g. Laeven and Goovaerts [13], Zaks et al. [20], Frostig et al. [10], Belles-Sampera [4], Bauer and Zanjani [2,3] and references therein.

In recent years, there has been growing interest in studying the optimal allocation problems because they play a central role in Solvency II. For example, Dhaene et al. [9] assumed a portfolio consisting of $n$ risks $X_{1}, X_{2}, \cdots, X_{n}$ and a company wished to allocate the total capital $d=d_{1}+d_{2}+\cdots+d_{n}$ to the corresponding risks. They formulated capital allocation as an optimization problem and provided a reasonable criterion, which is to set the amount $d_{i}$ to $X_{i}$ so that the potential loss measured by some appropriate

*School of Science, Nanchang Institute of Technology, Nanchang 330099, P.R.China

†School of Economics and Trade, Nanchang Institute of Technology, NanChang 330099, P.R.China

Email : jhxie@nit.edu.cn

$\ddagger$ Corresponding Author. 
distance measure is as small as possible. And further, they proposed to model the capital allocation problem by the following optimization problem:

$$
\min _{d_{1}, \cdots, d_{n}} \sum_{i=1}^{n} v_{i} \mathbf{E}\left[\zeta_{i} D\left(\frac{X_{i}-d_{i}}{v_{i}}\right)\right], \quad \text { such that } \sum_{i=1}^{n} d_{i}=d,
$$

where the $v_{i}, i=1,2, \cdots, n$, are non-negative real numbers such that $\sum_{i=1}^{n} v_{i}=1, D$ is a non-negative function and the $\zeta_{i}, i=1,2, \cdots, n$, are non-negative random variables such that $\mathbf{E}\left[\zeta_{i}\right]=1$. The non-negative real number $v_{i}$ is a measure of exposure or business volume of the $i$ th unit, such as revenue, insurance premium, etc. $D$ is a distance measurement function that measures the loss of allocation. The non-negative random variables $\zeta_{i}, i=1,2, \cdots, n$, are used as weight factors to the different possible outcomes of $D\left(X_{i}-d_{i}\right)$. The framework is general and includes many optimisation criterion as special cases, such as Quantile, Haircut and Covariance. A special case of (1.1) is the following optimization problem:

$$
\min _{d_{1}, \cdots, d_{n}} \sum_{i=1}^{n} v_{i} \mathbf{E}\left[\left(X_{i}-d_{i}\right)^{2}\right], \quad \text { such that } \sum_{i=1}^{n} d_{i}=d,
$$

which is showed in Dhaene et al. [9]. The solution to the equation above is

$$
d_{i}=\frac{d}{n}+\mathbf{E}\left(X_{i}\right)-\frac{1}{n} \sum_{j=1}^{n} \mathbf{E}\left(X_{j}\right), \quad i=1,2, \cdots, n
$$

$\mathrm{Xu}$ and $\mathrm{Hu}[17]$ generalized this idea and defined the following loss function:

$$
L(\vec{p})=\sum_{i=1}^{n} D\left(X_{i}-p_{i}\right)
$$

Then, they proposed to model the capital allocation problem by the following optimization problem:

$$
\min _{\vec{p} \in A} \operatorname{Pr}(L(\vec{p}) \geq t), \quad \forall t \geq 0 .
$$

You and $\mathrm{Li}$ [19] further studied the capital allocation concerning mutually exchangeable random risks. Cai and Wei [5] proposed some new notions of dependence with applications in optimal allocation problems. Zaks and Tsanakas [21] studied the optimal capital allocation in a hierarchical corporate structure where stake holders at two organizational levels. Manesh and Khaledi [14] considered the allocations of policy limits and ordering relations for aggregate remaining claims.

Note that allocation criterion based on minimizing the loss function does not take into account the the factor of variability of loss function. We also note that both allocation rule (1.1) and (1.2) do not take into account tail risk. Xu and Mao [18] proposed a Tail Mean-Variance (TMV) framework to overcome this limitation. In more detail, they provided the following allocation rule:

$$
\begin{gathered}
\min _{d_{1}, \cdots, d_{n}}\left\{\mathbf{E}\left[\sum_{i=1}^{n}\left(X_{i}-d_{i}\right)^{2} \mid S>\operatorname{VaR}_{\kappa}(S)\right]+\beta \operatorname{Var}\left(\sum_{i=1}^{n}\left(X_{i}-d_{i}\right)^{2} \mid S>\operatorname{VaR}_{\kappa}(S)\right)\right\} \\
\text { such that } \sum_{i=1}^{n} d_{i}=d
\end{gathered}
$$

where $\beta>0, S=\sum_{i=1}^{n} X_{i}$ is the aggregate risk, $\operatorname{VaR}_{\kappa}(S)=\inf (x \in \mathbf{R}, F(S) \geq \kappa)$ is the value at risk at level $\kappa, 0<\kappa<1$ of $S$ and $F(S)=\operatorname{Pr}(S \leq s)$ is the distribution of $S$. $\mathrm{Xu}$ and Mao [18] studied the optimal capital allocations when the risks have multivariate elliptical distributions. 
In most papers described above, the dependence between different lines of business of a portfolio is due to construction of a multivariate distribution. Copulas are currently seen as flexible and effective tools to describe dependence between the random variables. In this paper, we propose introducing dependence with a copula. We use the FarlieGumbel-Morgenstern (FGM) copula, like Bargès et al. [1] and Cossette et al. [7], to describe the dependence. Based on the simplicity and tractability of FGM copula, we derive the closed-form expressions for the optimal capital allocations based on the Tail Mean-Variance model when the FGM copula represent dependence between the risk marginals.

The work of this paper can be seen as a complement to the research on the the optimal capital allocations based on the Tail Mean-Variance principle and extend the former results by introducing dependence with a copula. When the optimal capital allocation problems for a portfolio consisting of different lines of risks linked by a Farlie-GumbelMorgenstern copula are considered, we show that, the explicit capital allocation formulae can be obtained under the Tail Mean-Variance principle.

The rest of the paper is organized as follows. In Section 2, we describe basic definitions and properties of the FGM copula and the exponential distributions. In Section 3, we derive explicit capital allocation formulae for the bivariate case, where both losses are exponentially distributed with the dependence linked by the FGM copula. In Section 4, we extend the results to the multivariate case. Some numerical examples to calculate the optimal capital allocation are presented to illustrate the solution procedure in Section 5 .

\section{Definitions}

Here, we briefly recall the characteristics and definition of the FGM copula. The FGM copula is a perturbation of the independence copula and it is not Archimedian. The FGM copula is a first order approximation of the Plackett copula (Nelsen [15], p.100) and of the Frank copula (p.133). Among the recent applications of the FGM copula, we mention Bargès et al. [1] and Cossette et al. [7] who deal with the application of the TVaR-based allocation rule using the FGM copula. The FGM copula is applied in the context of risk measurement by Gebizlioglu and Yagci [11] and in the context of sums of dependent r.v. by Geluk and Tang [12]. The FGM copula is also used in a ruin context by Cossette et al. [8], Xie and Zou [16]. The FGM copula is attractive due to its tractability and its simplicity.

We assume the lines of business of an portfolio are linked with a FGM copula. Since the exponential distribution is a classic distribution for the risk random variables and its practical mathematic properties allow explicit results, we assume marginal risks are distributed as exponentials.

\subsection{The bivariate case}

Firstly, we consider two business lines whose losses $X_{1}$ and $X_{2}$ follow the exponential distribution: $X_{i} \sim \operatorname{Exp}\left(\lambda_{i}\right), i=1,2$. Their probability density functions (pdf) $f_{X_{i}}$ and cumulative distribution functions (cdf) $F_{X_{i}}$ are given by

$$
f_{X_{i}}=\lambda_{i} e^{-\lambda_{i} x_{i}}, \quad F_{X_{i}}=1-e^{-\lambda_{i} x_{i}}, \quad \text { for } i=1,2 \text {. }
$$

We assume that the couple $\left(X_{1}, X_{2}\right)$ has a bivariate distribution defined with FGM copula. The FGM copula is defined as follows:

$$
C_{\theta}^{F G M}\left(u_{1}, u_{2}\right)=u_{1} u_{2}+\theta u_{1} u_{2}\left(1-u_{1}\right)\left(1-u_{2}\right),
$$


for $\left(u_{1}, u_{2}\right) \in[0,1] \times[0,1]$. The dependence parameter $\theta$ takes value in $[-1,1]$, where $\theta<0(>0)$ corresponds to a negative (positive) dependence relation. The density of the bivariate FGM copula is

$$
c_{\theta}^{F G M}\left(u_{1}, u_{2}\right)=\frac{\partial^{2} C_{\theta}^{F G M}\left(u_{1}, u_{2}\right)}{\partial u_{1} \partial u_{2}}=1+\theta\left(2 \bar{u}_{1}-1\right)\left(2 \bar{u}_{2}-1\right),
$$

where $\bar{u}_{i}=1-u_{i}, i=1,2$. Then, the joint c.d.f. $F_{X_{1}, X_{2}}\left(x_{1}, x_{2}\right)$ of $\left(X_{1}, X_{2}\right)$ with marginals $F_{X_{1}}$ and $F_{X_{2}}$ and defined with the FGM copula is given by

$$
\begin{aligned}
F_{X_{1}, X_{2}}\left(x_{1}, x_{2}\right) & =C_{\theta}^{F G M}\left(F_{X_{1}}\left(x_{1}\right), F_{X_{2}}\left(x_{2}\right)\right) \\
& =F_{X_{1}}\left(x_{1}\right) F_{X_{2}}\left(x_{2}\right)+\theta F_{X_{1}}\left(x_{1}\right) F_{X_{2}}\left(x_{2}\right)\left(1-F_{X_{1}}\left(x_{1}\right)\right)\left(1-F_{X_{2}}\left(x_{2}\right)\right) .
\end{aligned}
$$

The joint pdf of $\left(X_{1}, X_{2}\right)$ is

$$
\begin{aligned}
f_{X_{1}, X_{2}}\left(x_{1}, x_{2}\right)= & c_{\theta}^{F G M}\left(F_{X_{1}}\left(x_{1}\right), F_{X_{2}}\left(x_{2}\right)\right) f_{X_{1}}\left(x_{1}\right) f_{X_{2}}\left(x_{2}\right) \\
= & (1+\theta) \lambda_{1} e^{-\lambda_{1} x_{1}} \lambda_{2} e^{-\lambda_{2} x_{2}}-\theta 2 \lambda_{1} e^{-2 \lambda_{1} x_{1}} \lambda_{2} e^{-\lambda_{2} x_{2}} \\
& -\theta \lambda_{1} e^{-\lambda_{1} x_{1}} 2 \lambda_{2} e^{-2 \lambda_{2} x_{2}}+\theta 2 \lambda_{1} e^{-2 \lambda_{1} x_{1}} 2 \lambda_{2} e^{-2 \lambda_{2} x_{2}} .
\end{aligned}
$$

\subsection{The multivariate case}

Now, we consider $n$ business lines whose losses $X_{1}, X_{2}, \cdots, X_{n}$ follow the exponential distribution: $X_{i} \sim \operatorname{Exp}\left(\lambda_{i}\right), i=1,2, \cdots, n$. We also assume that the $n$ different risks jointed by a multivariate FGM $n$-copula, which has $2^{n}-n-1$ parameters, is defined as follows:

$$
C_{\theta}^{F G M}\left(u_{1}, \ldots, u_{n}\right)=u_{1} \cdots u_{n}\left(1+\sum_{q=2}^{n} \sum_{1 \leq p_{1}<\cdots<p_{q} \leq n} \theta_{p_{1} \ldots p_{q}} \bar{u}_{p_{1}} \cdots \bar{u}_{p_{q}}\right),
$$

where $\theta \in[-1,1], \bar{u}_{i}=1-u_{i}, i=1,2, \cdots, n$. Its density can be written as

$$
c_{\theta}^{F G M}\left(u_{1}, \ldots, u_{n}\right)=1+\sum_{q=2}^{n} \sum_{1 \leq p_{1}<\cdots<p_{q} \leq n} \theta_{p_{1} \cdots p_{q}}\left(2 \bar{u}_{p_{1}}-1\right) \cdots\left(2 \bar{u}_{p_{q}}-1\right) .
$$

Then, the joint pdf of $\left(X_{1}, \ldots, X_{n}\right)$ is

$$
\begin{aligned}
& f_{X_{1}, \ldots, X_{n}}\left(x_{1}, \ldots, x_{n}\right)=c_{\theta}^{F G M}\left(F_{X_{1}}\left(x_{1}\right), \ldots, F_{X_{n}}\left(x_{n}\right)\right) f_{X_{1}}\left(x_{1}\right) \cdots f_{X_{n}}\left(x_{n}\right) \\
& =f_{X_{1}}\left(x_{1}\right) \cdots f_{X_{n}}\left(x_{n}\right)\left(1+\sum_{q=2}^{n} \sum_{1 \leq p_{1}<\cdots<p_{q} \leq n} \theta_{p_{1} \cdots p_{q}}\left(1-F_{X_{p_{1}}}\left(x_{p_{1}}\right)\right) \cdots\left(1-F_{X_{p_{q}}}\left(x_{p_{q}}\right)\right)\right) \\
& =\omega\left(x_{1}, \ldots, x_{n} ; \lambda_{1}, \ldots, \lambda_{n}\right)+\sum_{q=2}^{n} \sum_{1 \leq p_{1}<\cdots<p_{q} \leq n} \theta_{p_{1} \cdots p_{q}} \times \\
& \left(\sum_{l=0}^{q} \sum_{\left(\epsilon_{1}, \ldots, \epsilon_{q}\right) \in \tau_{l, q}}(-1)^{l} \omega\left(x_{p_{1}}, \ldots, x_{p_{q}} ; x_{i_{q+1}}, \ldots, x_{i_{n}} ; 2^{\epsilon} \lambda_{p_{1}}, \ldots, 2^{\epsilon} \lambda_{p_{q}}, \lambda_{i_{q+1}}, \cdots, \lambda_{i_{n}}\right)\right),
\end{aligned}
$$

where $\omega\left(x_{1}, x_{2}, \cdots, x_{n} ; \alpha_{1}, \alpha_{2}, \cdots, \alpha_{n}\right)=\alpha_{1} e^{-\alpha_{1} x_{1}} \alpha_{2} e^{-\alpha_{2} x_{2}} \cdots \alpha_{n} e^{-\alpha_{n} x_{n}}, i_{q+1}, \cdots, i_{n}$ are the missing indexes of $p_{1}, \cdots, p_{q}$ to complete $1, \cdots, n$ and $\tau_{l, q}$ are the sets of $q$ tuples composed of $l$ zeros and $q-l$ ones, i.e., $\tau_{l, q}, l=0,1, \cdots, q$ and $q=2, \cdots, n$ are defined as $\tau_{0, q}=\left\{(1,1, \cdots, 1)_{1 \times q}\right\}, \tau_{1, q}=\left\{(1,1, \cdots, 0)_{1 \times q}, \cdots,(0,1, \cdots, 1)_{1 \times q}\right\}$, $\tau_{2, q}=\left\{(1,1, \cdots, 0,0)_{1 \times q}, \cdots,(0,0, \cdots, 1)_{1 \times q}\right\}, \cdots, \tau_{q, q}=\left\{(0,0, \cdots, 0)_{1 \times q}\right\}$. 


\section{Calculation of the optimal capital allocation for two risks}

In this section, we obtain the close formed expression of the optimal capital allocation based on the Tail Mean-Variance (TMV) model for two exponentially distributed risks linked by a FGM copula. Let $X_{1}$ and $X_{2}$ are two exponentially distributed with $\lambda_{1}$ and $\lambda_{2}$. In order to simplify the calculation, we assume that $\lambda_{1} \neq \lambda_{2}, \lambda_{1} \neq 2 \lambda_{2}$, and $\lambda_{2} \neq 2 \lambda_{1}$. Applying the similar technique proposed below, one can obtains the adjusted results without these restrictions.

Theorem 3.1. Let $X_{1}$ and $X_{2}$ are two exponentially distributed with $\lambda_{1}$ and $\lambda_{2}$. The dependence structure for $\left(X_{1}, X_{2}\right)$ is defined by the bivariate FGM copula with parameter $\theta \in[-1,1]$. Then the optimal allocation solution $\vec{d}^{*}=\left(d_{1}^{*}, d_{2}^{*}\right)$ to the TMV model

$$
\begin{gathered}
\min _{d_{1}, d_{2}}\left\{\mathbf{E}\left[\sum_{i=1}^{2}\left(X_{i}-d_{i}\right)^{2} \mid S>\operatorname{VaR}_{\kappa}(S)\right]+\beta \operatorname{Var}\left(\sum_{i=1}^{2}\left(X_{i}-d_{i}\right)^{2} \mid S>\operatorname{VaR}_{\kappa}(S)\right)\right\}, \\
\text { such that } d_{1}+d_{2}=d,
\end{gathered}
$$

is presented as follows:

$$
\begin{aligned}
d_{1}= & \frac{1}{2\left(1-2 \beta\left(2 E^{X_{1} X_{2}}+\left(E^{X_{1}}-E^{X_{2}}\right)^{2}-\left(E^{X_{1}^{2}}+E^{X_{2}^{2}}\right)\right)\right)} \times \\
& \left(2 \beta\left(E^{X_{1} X_{2}^{2}}-E^{X_{1}^{2} X_{2}}+E^{X_{1}^{3}}-E^{X_{2}^{3}}\right)+\left(E^{X_{1}}-E^{X_{2}}\right)\left(1-2 \beta\left(E^{X_{1}^{2}}+E^{X_{2}^{2}}\right)\right)\right. \\
& \left.+d\left(1-4 \beta\left(E^{X_{1} X_{2}}-E^{X_{2}^{2}}+E^{X_{2}}\left(E^{X_{2}}-E^{X_{1}}\right)\right)\right)\right) \\
d_{2}= & \frac{1}{2\left(1-2 \beta\left(2 E^{X_{1} X_{2}}+\left(E^{X_{1}}-E^{X_{2}}\right)^{2}-\left(E^{X_{1}^{2}}+E^{X_{2}^{2}}\right)\right)\right)} \times \\
& \left(-2 \beta\left(E^{X_{1} X_{2}^{2}}-E^{X_{1}^{2} X_{2}}+E^{X_{1}^{3}}-E^{X_{2}^{3}}\right)-\left(E^{X_{1}}-E^{X_{2}}\right)\left(1-2 \beta\left(E^{X_{1}^{2}}+E^{X_{2}^{2}}\right)\right)\right. \\
& \left.+d\left(1-4 \beta\left(E^{X_{1} X_{2}}-E^{X_{1}^{2}}+E^{X_{1}}\left(E^{X_{1}}-E^{X_{2}}\right)\right)\right)\right)
\end{aligned}
$$

where

$$
\begin{gathered}
E^{X_{i}^{k}}=\mathbf{E}\left[X_{i}^{k} \mid S>\operatorname{VaR}_{\kappa}(S)\right]=\frac{1}{1-\kappa}\left[(1+\theta) \xi_{k}\left(\operatorname{VaR}_{\kappa}(S) ; \lambda_{i} ; \lambda_{j}\right)-\theta \xi_{k}\left(\operatorname{VaR}_{\kappa}(S) ; 2 \lambda_{i} ; \lambda_{j}\right)\right. \\
\left.-\theta \xi_{k}\left(\operatorname{VaR}_{\kappa}(S) ; \lambda_{i} ; 2 \lambda_{j}\right)+\theta \xi_{k}\left(\operatorname{VaR}_{\kappa}(S) ; 2 \lambda_{i} ; 2 \lambda_{j}\right)\right], \quad i, j=1,2, \quad i \neq j, \quad k=1,2,3, \\
E^{X_{i}^{2} X_{j}}=\mathbf{E}\left[X_{i}^{2} X_{j} \mid S>\operatorname{VaR}_{\kappa}(S)\right]=\frac{1}{1-\kappa}\left[(1+\theta) \chi\left(\operatorname{VaR}_{\kappa}(S) ; \lambda_{i} ; \lambda_{j}\right)\right. \\
\left.-\theta \chi\left(\operatorname{VaR}_{\kappa}(S) ; 2 \lambda_{i} ; \lambda_{j}\right)-\theta \chi\left(\operatorname{VaR}_{\kappa}(S) ; \lambda_{i} ; 2 \lambda_{j}\right)+\theta \chi\left(\operatorname{VaR}_{\kappa}(S) ; 2 \lambda_{i} ; 2 \lambda_{j}\right)\right], i, j=1,2, i \neq j, \\
E^{X_{i} X_{j}}=\mathbf{E}\left[X_{i} X_{j} \mid S>\operatorname{VaR}_{\kappa}(S)\right]=\frac{1}{1-\kappa}\left[(1+\theta) \varphi\left(\operatorname{VaR}_{\kappa}(S) ; \lambda_{i} ; \lambda_{j}\right)\right. \\
\left.-\theta \varphi\left(\operatorname{VaR}_{\kappa}(S) ; 2 \lambda_{i} ; \lambda_{j}\right)-\theta \varphi\left(\operatorname{VaR}_{\kappa}(S) ; \lambda_{i} ; 2 \lambda_{j}\right)+\theta \varphi\left(\operatorname{VaR}_{\kappa}(S) ; 2 \lambda_{i} ; 2 \lambda_{j}\right)\right], \\
\xi_{1}\left(x ; \alpha_{i} ; \alpha_{j}\right)=\frac{\alpha_{j} e^{-\alpha_{i} x}\left(x+\frac{1}{\alpha_{i}}\right)}{\alpha_{j}-\alpha_{i}}-\frac{\alpha_{j} e^{-\alpha_{i} x}-\alpha_{i} e^{-\alpha_{j} x}}{\left(\alpha_{j}-\alpha_{i}\right)^{2}}, \\
\xi_{2}\left(x ; \alpha_{i} ; \alpha_{j}\right)=\frac{e^{-\alpha_{i} x} \alpha_{j}\left(\frac{2}{\alpha_{i}^{2}}+\frac{2 x}{\alpha_{i}}+x^{2}\right)}{\alpha_{j}-\alpha_{i}}-\frac{2 \alpha_{j} e^{-\alpha_{i} x}\left(x+\frac{1}{\alpha_{i}}\right)}{\left(\alpha_{j}-\alpha_{i}\right)^{2}}+\frac{2\left(\alpha_{j} e^{-\alpha_{i} x}-\alpha_{i} e^{-\alpha_{j} x}\right)}{\left(\alpha_{j}-\alpha_{i}\right)^{3}}, \\
\xi_{3}\left(x ; \alpha_{i} ; \alpha_{j}\right)=\frac{e^{-\alpha_{i} x} \alpha_{j}\left(\frac{6}{\alpha_{i}^{3}}+\frac{6 x}{\alpha_{i}^{2}}+\frac{3 x^{2}}{\alpha_{i}}+x^{3}\right)}{\alpha_{j}-\alpha_{i}}-\frac{3 e^{-\alpha_{i} x} \alpha_{j}\left(\frac{2}{\alpha_{i}^{2}}+\frac{2 x}{\alpha_{i}}+x^{2}\right)}{\left(\alpha_{j}-\alpha_{i}\right)^{2}} \\
+\frac{6 \alpha_{j} e^{-\alpha_{i} x}\left(x+\frac{1}{\alpha_{i}}\right)}{\left(\alpha_{j}-\alpha_{i}\right)^{3}}-\frac{6\left(\alpha_{j} e^{-\alpha_{i} x}-\alpha_{i} e^{-\alpha_{j} x}\right)}{\left(\alpha_{j}-\alpha_{i}\right)^{4}},
\end{gathered}
$$




$$
\begin{aligned}
\chi\left(x ; \alpha_{i} ; \alpha_{j}\right) & =\frac{\alpha_{j} e^{-\alpha_{i} x}\left(\frac{2}{\alpha_{i}^{2}}+\frac{2 x}{\alpha_{i}}+x^{2}\right)}{\left(\alpha_{j}-\alpha_{i}\right)^{2}}-\frac{4 \alpha_{j} e^{-\alpha_{i} x}\left(x+\frac{1}{\alpha_{i}}\right)+2 \alpha_{i} e^{-\alpha_{j} x}\left(x+\frac{1}{\alpha_{j}}\right)}{\left(\alpha_{j}-\alpha_{i}\right)^{3}} \\
+\frac{6\left(\alpha_{j} e^{-\alpha_{i} x}-\alpha_{i} e^{-\alpha_{j} x}\right)}{\left(\alpha_{j}-\alpha_{i}\right)^{4}}, & \\
\varphi\left(x ; \alpha_{i} ; \alpha_{j}\right) & =-\frac{\alpha_{j} e^{-\alpha_{i} x}\left(x+\frac{1}{\alpha_{i}}\right)+\alpha_{i} e^{-\alpha_{j} x}\left(x+\frac{1}{\alpha_{j}}\right)}{\left(\alpha_{j}-\alpha_{i}\right)^{2}}+\frac{2\left(\alpha_{j} e^{-\alpha_{i} x}-\alpha_{i} e^{-\alpha_{j} x}\right)}{\left(\alpha_{j}-\alpha_{i}\right)^{3}} .
\end{aligned}
$$

Proof. In order to find the optimal allocation solution $\vec{d}^{*}=\left(d_{1}^{*}, d_{2}^{*}\right)$, we utilize the method of Lagrange multiplier. Assume

$$
\begin{aligned}
l\left(d_{1}, d_{2}\right)= & \mathbf{E}\left[\left(X_{1}-d_{1}\right)^{2}+\left(X_{2}-d_{2}\right)^{2} \mid S>\operatorname{VaR}_{\kappa}(S)\right] \\
& +\beta \operatorname{Var}\left(\left(X_{1}-d_{1}\right)^{2}+\left(X_{2}-d_{2}\right)^{2} \mid S>\operatorname{VaR}_{\kappa}(S)\right),
\end{aligned}
$$

and

$$
L\left(d_{1}, d_{2}, \gamma\right)=l\left(d_{1}, d_{2}\right)+\gamma\left(d-d_{1}-d_{2}\right)
$$

Since

$$
\begin{aligned}
& \operatorname{Var}\left(\left(X_{1}-d_{1}\right)^{2}+\left(X_{2}-d_{2}\right)^{2} \mid S>\operatorname{VaR}_{\kappa}(S)\right) \\
= & \mathbf{E}\left[\left(\left(X_{1}\right)^{2}+\left(X_{2}\right)^{2}-2 d_{2} X_{2}\right)^{2} \mid S>\operatorname{VaR}_{\kappa}(S)\right]+\left(E^{X_{1}^{2}}+E^{X_{2}^{2}}-2 d_{2} E^{X_{2}}\right)^{2}+4 d_{1}^{2}\left(E^{X_{1}^{2}}\right. \\
- & \left.\left(E^{X_{1}}\right)^{2}\right)+4 d_{1}\left[2 d_{2}\left(E^{X_{1} X_{2}}-E^{X_{1}} E^{X_{2}}\right)-\left(E^{X_{1}^{3}}-E^{X_{1}} E^{X_{1}^{2}}\right)-\left(E^{X_{1} X_{2}^{2}}-E^{X_{1}} E^{X_{2}^{2}}\right)\right],
\end{aligned}
$$

we get

$$
\begin{aligned}
& \frac{\partial L\left(d_{1}, d_{2}, \gamma\right)}{\partial d_{1}}=-2 E^{X_{1}}+2 d_{1}+8 \beta d_{1}\left(E^{X_{1}^{2}}-\left(E^{X_{1}}\right)^{2}\right) \\
& +4 \beta\left[2 d_{2}\left(E^{X_{1} X_{2}}-E^{X_{1}} E^{X_{2}}\right)-\left(E^{X_{1}^{3}}-E^{X_{1}} E^{X_{1}^{2}}\right)-\left(E^{X_{1} X_{2}^{2}}-E^{X_{1}} E^{X_{2}^{2}}\right)\right]-\gamma=0 .
\end{aligned}
$$

Similarly,

$$
\begin{aligned}
& \frac{\partial L\left(d_{1}, d_{2}, \gamma\right)}{\partial d_{2}}=-2 E^{X_{2}}+2 d_{2}+8 \beta d_{2}\left(E^{X_{2}^{2}}-\left(E^{X_{2}}\right)^{2}\right) \\
& +4 \beta\left[2 d_{1}\left(E^{X_{1} X_{2}}-E^{X_{1}} E^{X_{2}}\right)-\left(E^{X_{2}^{3}}-E^{X_{2}} E^{X_{2}^{2}}\right)-\left(E^{X_{2} X_{1}^{2}}-E^{X_{2}} E^{X_{1}^{2}}\right)\right]-\gamma=0,
\end{aligned}
$$

and

$$
\frac{\partial L\left(d_{1}, d_{2}, \gamma\right)}{\partial \gamma}=d_{1}+d_{2}-d=0 .
$$

From Eqs.(3.3)-(3.5), the results of (3.1) and (3.2) follow immediately. Now, we need to find the formulae of $E^{X_{i}^{k}}, E^{X_{i}^{2} X_{j}}$ and $E^{X_{1} X_{2}}$. Firstly, the explicit expression of $E^{X_{1}^{3}}$ is calculated as follow.

$$
\begin{aligned}
E^{X_{1}^{3}} & =\mathbf{E}\left[X_{1}^{3} \mid S>\operatorname{VaR}_{\kappa}(S)\right]=\int_{\operatorname{VaR}_{\kappa}(S)}^{\infty} \mathbf{E}\left[X_{1}^{3} \mid S=s\right] f_{S \mid S>\operatorname{VaR}_{\kappa}(S)}(s) \mathrm{d} s \\
& =\frac{\int_{\operatorname{VaR}_{\kappa}(S)}^{\infty} \mathbf{E}\left[X_{1}^{3} \mid S=s\right] f_{S}(s) \mathrm{d} s}{\operatorname{Pr}\left(S>\operatorname{VaR}_{\kappa}(S)\right)}=\frac{\int_{\operatorname{VaR}_{\kappa}(S)}^{\infty} f_{S}(s) \int_{0}^{s} x^{3} f_{X_{1} \mid S}(x \mid S=s) \mathrm{d} x \mathrm{~d} s}{1-F_{S}\left(\operatorname{VaR}_{\kappa}(S)\right)} \\
& =\frac{\int_{\operatorname{VaR}_{\kappa}(S)}^{\infty} \int_{0}^{s} x^{3} f_{x_{1}, S}(x, s) \mathrm{d} x \mathrm{~d} s}{1-\kappa} .
\end{aligned}
$$

Note that $S=X_{1}+X_{2}$. From Eq.(2.1), we get

$$
\begin{aligned}
& \int_{0}^{s} x^{3} f_{x_{1}, S}(x, s) \mathrm{d} x=\int_{0}^{s} x^{3} f_{x_{1}, x_{2}}(x, s-x) \mathrm{d} x \\
& \quad=(1+\theta) \lambda_{1} \lambda_{2}\left(\frac{6\left(e^{-\lambda_{2} s}-e^{-\lambda_{1} s}\right)}{\left(\lambda_{2}-\lambda_{1}\right)^{4}}+\frac{6 s e^{-\lambda_{1} s}}{\left(\lambda_{2}-\lambda_{1}\right)^{3}}-\frac{3 s^{2} e^{-\lambda_{1} s}}{\left(\lambda_{2}-\lambda_{1}\right)^{2}}+\frac{s^{3} e^{-\lambda_{1} s}}{\lambda_{2}-\lambda_{1}}\right)
\end{aligned}
$$




$$
\begin{gathered}
-\theta 2 \lambda_{1} \lambda_{2}\left(\frac{6\left(e^{-\lambda_{2} s}-e^{-2 \lambda_{1} s}\right)}{\left(\lambda_{2}-2 \lambda_{1}\right)^{4}}+\frac{6 s e^{-2 \lambda_{1} s}}{\left(\lambda_{2}-2 \lambda_{1}\right)^{3}}-\frac{3 s^{2} e^{-2 \lambda_{1} s}}{\left(\lambda_{2}-2 \lambda_{1}\right)^{2}}+\frac{s^{3} e^{-2 \lambda_{1} s}}{\lambda_{2}-2 \lambda_{1}}\right) \\
-\theta \lambda_{1} 2 \lambda_{2}\left(\frac{6\left(e^{-2 \lambda_{2} s}-e^{-\lambda_{1} s}\right)}{\left(2 \lambda_{2}-\lambda_{1}\right)^{4}}+\frac{6 s e^{-\lambda_{1} s}}{\left(2 \lambda_{2}-\lambda_{1}\right)^{3}}-\frac{3 s^{2} e^{-\lambda_{1} s}}{\left(2 \lambda_{2}-\lambda_{1}\right)^{2}}+\frac{s^{3} e^{-\lambda_{1} s}}{2 \lambda_{2}-\lambda_{1}}\right) \\
+\theta 2 \lambda_{1} 2 \lambda_{2}\left(\frac{6\left(e^{-2 \lambda_{2} s}-e^{-2 \lambda_{1} s}\right)}{\left(2 \lambda_{2}-2 \lambda_{1}\right)^{4}}+\frac{6 s e^{-2 \lambda_{1} s}}{\left(2 \lambda_{2}-2 \lambda_{1}\right)^{3}}-\frac{3 s^{2} e^{-2 \lambda_{1} s}}{\left(2 \lambda_{2}-2 \lambda_{1}\right)^{2}}+\frac{s^{3} e^{-2 \lambda_{1} s}}{2 \lambda_{2}-2 \lambda_{1}}\right) .
\end{gathered}
$$

Define

$$
\begin{aligned}
\lambda_{1} \lambda_{2} \int_{\operatorname{VaR}_{\kappa}(S)}^{\infty}\left(\frac{6\left(e^{-\lambda_{2} s}-e^{-\lambda_{1} s}\right)}{\left(\lambda_{2}-\lambda_{1}\right)^{4}}+\frac{6 s e^{-\lambda_{1} s}}{\left(\lambda_{2}-\lambda_{1}\right)^{3}}-\frac{3 s^{2} e^{-\lambda_{1} s}}{\left(\lambda_{2}-\lambda_{1}\right)^{2}}+\frac{s^{3} e^{-\lambda_{1} s}}{\lambda_{2}-\lambda_{1}}\right) \mathrm{d} s \\
=\frac{e^{-\lambda_{1} \operatorname{VaR}_{\kappa}(S)} \lambda_{2}\left(\frac{6}{\lambda_{1}^{3}}+\frac{6 \operatorname{VaR}_{\kappa}(S)}{\lambda_{1}^{2}}+\frac{3\left(\operatorname{VaR}_{\kappa}(S)\right)^{2}}{\lambda_{1}}+\left(\operatorname{VaR}_{\kappa}(S)\right)^{3}\right)}{\lambda_{2}-\lambda_{1}} \\
\quad-\frac{3 e^{-\lambda_{1} \operatorname{VaR}_{\kappa}(S)} \lambda_{2}\left(\frac{2}{\lambda_{1}^{2}}+\frac{2 \operatorname{VaR}_{\kappa}(S)}{\lambda_{1}}+\left(\operatorname{VaR}_{\kappa}(S)\right)^{2}\right)}{\left(\lambda_{2}-\lambda_{1}\right)^{2}} \\
\quad+\frac{6 \lambda_{2} e^{-\lambda_{1} \operatorname{VaR}_{\kappa}(S)}\left(\operatorname{VaR}_{\kappa}(S)+\frac{1}{\lambda_{1}}\right)}{\left(\lambda_{2}-\lambda_{1}\right)^{3}}-\frac{6\left(\lambda_{2} e^{-\lambda_{1} \operatorname{VaR}_{\kappa}(S)}-\lambda_{1} e^{-\lambda_{2} \operatorname{VaR}_{\kappa}(S)}\right)}{\left(\lambda_{2}-\lambda_{1}\right)^{4}} \\
=\xi_{3}\left(\operatorname{VaR}_{\kappa}(S) ; \lambda_{1} ; \lambda_{2}\right) .
\end{aligned}
$$

Plugging Eqs.(3.7) and (3.8) into Eq.(3.6), we can derive the formula of

$$
\begin{aligned}
E^{X_{1}^{3}}= & \frac{1}{1-\kappa}\left((1+\theta) \xi_{3}\left(\operatorname{VaR}_{\kappa}(S) ; \lambda_{1} ; \lambda_{2}\right)-\theta \xi_{3}\left(\operatorname{VaR}_{\kappa}(S) ; 2 \lambda_{1} ; \lambda_{2}\right)\right. \\
& \left.-\theta \xi_{3}\left(\operatorname{VaR}_{\kappa}(S) ; \lambda_{1} ; 2 \lambda_{2}\right)+\theta \xi_{3}\left(\operatorname{VaR}_{\kappa}(S) ; 2 \lambda_{1} ; 2 \lambda_{2}\right)\right)
\end{aligned}
$$

The formula of $E^{X_{2}^{3}}$ is symmetrically given by

$$
\begin{aligned}
E^{X_{2}^{3}}= & \frac{1}{1-\kappa}\left((1+\theta) \xi_{3}\left(\operatorname{VaR}_{\kappa}(S) ; \lambda_{2} ; \lambda_{1}\right)-\theta \xi_{3}\left(\operatorname{VaR}_{\kappa}(S) ; 2 \lambda_{2} ; \lambda_{1}\right)\right. \\
& \left.-\theta \xi_{3}\left(\operatorname{VaR}_{\kappa}(S) ; \lambda_{2} ; 2 \lambda_{1}\right)+\theta \xi_{3}\left(\operatorname{VaR}_{\kappa}(S) ; 2 \lambda_{1} ; 2 \lambda_{2}\right)\right) .
\end{aligned}
$$

Since the formulae of $E^{X_{i}}$ and $E^{X_{i}^{2}}$ can be calculated similarly, we omit the proof. Now, we give the formula of $E^{X_{1}^{2} X_{2}}$.

$$
\begin{aligned}
E^{X_{1}^{2} X_{2}}=\mathbf{E}\left[X_{1}^{2} X_{2} \mid S>\operatorname{VaR}_{\kappa}(S)\right]=\int_{\operatorname{VaR}_{\kappa}(S)}^{\infty} \mathbf{E}\left[X_{1}^{2} X_{2} \mid S=s\right] f_{S \mid S>\operatorname{VaR}_{\kappa}(S)}(s) \mathrm{d} s \\
=\int_{\operatorname{VaR}_{\kappa}(S)}^{\infty} \mathbf{E}\left[X_{1}^{2}\left(s-X_{1}\right) \mid S=s\right] f_{S \mid S>\operatorname{VaR}_{\kappa}(S)}(s) \mathrm{d} s \\
=\frac{\int_{\operatorname{VaR}_{\kappa}(S)}^{\infty} \mathbf{E}\left[X_{1}^{2}\left(s-X_{1}\right) \mid S=s\right] f_{S}(s) \mathrm{d} s}{\operatorname{Pr}\left(S>\operatorname{VaR}_{\kappa}(S)\right)}=\frac{\int_{\operatorname{VaR}_{\kappa}(S)}^{\infty} \int_{0}^{s} x^{2}(s-x) f_{x_{1}, S}(x, s) \mathrm{d} x \mathrm{~d} s}{1-\kappa} .
\end{aligned}
$$

Note that

$$
\int_{0}^{s} x^{2}(s-x) f_{x_{1}, S}(x, s) \mathrm{d} x=s \int_{0}^{s} x^{2} f_{x_{1}, x_{2}}(x, s-x) \mathrm{d} x-\int_{0}^{s} x^{3} f_{x_{1}, x_{2}}(x, s-x) \mathrm{d} x .
$$

By the similar calculations, we can derive

$$
\begin{aligned}
E^{X_{1}^{2} X_{2}}= & \frac{1}{1-\kappa}\left((1+\theta) \chi\left(\operatorname{VaR}_{\kappa}(S) ; \lambda_{1} ; \lambda_{2}\right)-\theta \chi\left(\operatorname{VaR}_{\kappa}(S) ; 2 \lambda_{1} ; \lambda_{2}\right)\right. \\
& \left.-\theta \chi\left(\operatorname{VaR}_{\kappa}(S) ; \lambda_{1} ; 2 \lambda_{2}\right)+\theta \chi\left(\operatorname{VaR}_{\kappa}(S) ; 2 \lambda_{1} ; 2 \lambda_{2}\right)\right) .
\end{aligned}
$$

The formula of $E^{X_{2}^{2} X_{1}}$ is also symmetrically given by

$$
\begin{aligned}
E^{X_{2}^{2} X_{1}}= & \frac{1}{1-\kappa}\left((1+\theta) \chi\left(\operatorname{VaR}_{\kappa}(S) ; \lambda_{2} ; \lambda_{1}\right)-\theta \chi\left(\operatorname{VaR}_{\kappa}(S) ; 2 \lambda_{2} ; \lambda_{1}\right)\right. \\
& \left.-\theta \chi\left(\operatorname{VaR}_{\kappa}(S) ; \lambda_{2} ; 2 \lambda_{1}\right)+\theta \chi\left(\operatorname{VaR}_{\kappa}(S) ; 2 \lambda_{2} ; 2 \lambda_{2}\right)\right) .
\end{aligned}
$$


Since the formula of $E^{X_{1} X_{2}}$ can be calculated similarly, we also omit the proof. This completes the proof of this theorem.

Remark 3.1. In Theorem 3.1, we assume that $\lambda_{1} \neq \lambda_{2}, \lambda_{1} \neq 2 \lambda_{2}$, and $\lambda_{2} \neq 2 \lambda_{1}$. In fact, one can obtains the adjusted results without these restrictions. Since the first part on the right-hand side of equation (3.6) can be rewritten as

$$
(1+\theta) \lambda_{1} \lambda_{2} e^{-\lambda_{1} s}\left(\frac{6\left(e^{\left(\lambda_{1}-\lambda_{2}\right) s}-1\right)}{\left(\lambda_{2}-\lambda_{1}\right)^{4}}+\frac{6 s}{\left(\lambda_{2}-\lambda_{1}\right)^{3}}-\frac{3 s^{2}}{\left(\lambda_{2}-\lambda_{1}\right)^{2}}+\frac{s^{3}}{\lambda_{2}-\lambda_{1}}\right),
$$

where $e^{\left(\lambda_{1}-\lambda_{2}\right) s}-1$ could be expanded in Talyor series if $\lambda_{2} \rightarrow \lambda_{1}$,

$$
e^{\left(\lambda_{1}-\lambda_{2}\right) s}-1=\left(\lambda_{1}-\lambda_{2}\right) s+\frac{\left(\lambda_{1}-\lambda_{2}\right)^{2} s^{2}}{2}+\frac{\left(\lambda_{1}-\lambda_{2}\right)^{3} s^{3}}{6}+\sum_{n=4}^{\infty} \frac{\left(\lambda_{1}-\lambda_{2}\right)^{n} s^{n}}{n !},
$$

then the limit of the first part on the right-hand side of equation (3.6) is

$$
\begin{gathered}
\lim _{\lambda_{2} \rightarrow \lambda_{1}}(1+\theta) \lambda_{1} \lambda_{2} e^{-\lambda_{1} s}\left(\frac{6\left(e^{\left(\lambda_{1}-\lambda_{2}\right) s}-1\right)}{\left(\lambda_{2}-\lambda_{1}\right)^{4}}+\frac{6 s}{\left(\lambda_{2}-\lambda_{1}\right)^{3}}-\frac{3 s^{2}}{\left(\lambda_{2}-\lambda_{1}\right)^{2}}+\frac{s^{3}}{\lambda_{2}-\lambda_{1}}\right) \\
=\lim _{\lambda_{2} \rightarrow \lambda_{1}} 6(1+\theta) \lambda_{1} \lambda_{2} e^{-\lambda_{1} s} \sum_{n=4}^{\infty} \frac{\left(\lambda_{1}-\lambda_{2}\right)^{n-4} s^{n}}{n !}=\frac{(1+\theta) \lambda_{1}^{2}}{4} e^{-\lambda_{1} s} s^{4} .
\end{gathered}
$$

For the case $\lambda_{1}=\lambda_{2}$, the first component of $f_{X_{1}, X_{2}}\left(x_{1}, x_{2}\right)$ can be rewritten as ( $1+$ $\theta) \lambda_{1}^{2} e^{-\lambda_{1}\left(x_{1}+x_{2}\right)}$, the first part on the right-hand side of equation (3.6) can be simplified as

$$
\int_{0}^{s} x^{3}(1+\theta) \lambda_{1}^{2} e^{-\lambda_{1}(x+(s-x))} \mathrm{d} x=\frac{(1+\theta) \lambda_{1}^{2}}{4} e^{-\lambda_{1} s} s^{4}
$$

By the results of (3.9) and (3.10), for the case $\lambda_{1}=\lambda_{2}$, we can apply the similar technique to obtain the adjusted results. For the cases $\lambda_{1}=2 \lambda_{2}$ and $\lambda_{2}=2 \lambda_{1}$, one obtains the similar results.

\section{Calculation of the optimal capital allocation for $n$ risks}

Explicit formulae for the optimal capital allocation for risks linked by a FGM copula cannot only be derived in the bivariate case but even for an undefined number of risks. In order to derive the explicit formulae for the optimal capital allocation, we first give the following lemma.

Lemma 4.1. Let $\vec{X}=\left(X_{1}, X_{2}, \cdots, X_{n}\right)$. Then the optimal allocation solution $\overrightarrow{d^{*}}=\left(d_{1}^{*}, d_{2}^{*}, \cdots, d_{n}^{*}\right)$ to the TMV model,

$$
\begin{gathered}
\min _{d_{1}, \cdots, d_{n}}\left\{\mathbf{E}\left[\sum_{i=1}^{n}\left(X_{i}-d_{i}\right)^{2} \mid S>\operatorname{VaR}_{\kappa}(S)\right]+\beta \operatorname{Var}\left(\sum_{i=1}^{n}\left(X_{i}-d_{i}\right)^{2} \mid S>\operatorname{VaR}_{\kappa}(S)\right)\right\}, \\
\text { such that } \sum_{i=1}^{n} d_{i}=d,
\end{gathered}
$$

is presented as follows:

$$
\vec{d}^{*}=\mathbf{A}^{-1} \vec{z}
$$

where $\mathbf{A}^{-1}=\left(a_{i j}\right)_{n \times n}$ is the inverse matrix of $\mathbf{A}=8 \beta \Sigma_{S}+2 \mathbf{I}_{n}, \Sigma_{S}$ is the conditional covariance matrix of $\left(\vec{X} \mid S>\operatorname{VaR}_{\kappa}(S)\right)$, and $\vec{z}=\left(\Lambda+\delta_{1}, \Lambda+\delta_{2}, \cdots, \Lambda+\delta_{n}\right)$ with $\delta_{i}=4 \beta \sum_{j=1}^{n} \operatorname{Cov}\left(X_{j}^{2}, X_{i} \mid S>\operatorname{VaR}_{\kappa}(S)\right)+2 \mathbf{E}\left[X_{i} \mid S>\operatorname{VaR}_{\kappa}(S)\right], i=1,2, \cdots, n$, where $\Lambda=\left(d-\sum_{i=1}^{n} \sum_{j=1}^{n} a_{i j} \delta_{j}\right) /\left(\sum_{i=1}^{n} \sum_{j=1}^{n} a_{i j}\right)$. 
Proof. The idea of proof is also based on the method of Lagrange multiplier. One can refer to $\mathrm{Xu}$ and Mao [17] for the full proof.

Note that Lemma 4.1 provides general formulae for optimal allocation based on the TMV model. There are some quantities, such as $\mathbf{E}\left[X_{i} \mid S>\operatorname{Var}_{\kappa}(S)\right], \operatorname{Cov}\left(X_{j}^{2}, X_{i} \mid S>\right.$ $\operatorname{VaR}_{\kappa}(S)$ ), and $\Sigma_{S}$, which are non-trivial to calculate. In order to find $\mathbf{E}\left[X_{i} \mid S>\operatorname{Var}_{\kappa}(S)\right]$, $\operatorname{Cov}\left(X_{j}^{2}, X_{i} \mid S>\operatorname{VaR}_{\kappa}(S)\right)$, and $\Sigma_{S}$, for the sake of deriving the explicit formulae for the optimal capital allocation, we need to derive the key quantities $E^{X_{i}^{k}}=\mathbf{E}\left[X_{i}^{k} \mid S>\right.$ $\left.\operatorname{VaR}_{\kappa}(S)\right], E^{X_{i}^{2} X_{j}}=\mathbf{E}\left[X_{i}^{2} X_{j} \mid S>\operatorname{VaR}_{\kappa}(S)\right]$, and $E^{X_{i} X_{j}}=\mathbf{E}\left[X_{i} X_{j} \mid S>\operatorname{VaR}_{\kappa}(S)\right]$, $k=1,2,3, i, j=1,2, \cdots, n, i \neq j$.

As in the bivariate case, the explicit formulae for the optimal capital allocation can also be explicitly given. In order to find the key quantities, we first introduce the $n$th order divided difference of the function $f$ presented in Chiragiev and Landsman [6]. Assume that $x_{1}, x_{2}, \cdots, x_{n}, x_{n+1}$ are arbitrary points and $x_{i} \neq x_{j}$ for $i \neq j$. The $n$th order divided difference of the function $f$ on $x_{1}, x_{2}, \cdots, x_{n}, x_{n+1}$ is defined as

$$
f\left(x_{1}, x_{2}, \cdots, x_{n}, x_{n+1}\right)=\sum_{i=1}^{n+1} \frac{f\left(x_{i}\right)}{\prod_{j \neq i}\left(x_{i}-x_{j}\right)} .
$$

In the follow proposition, we provide the explicit formulae of $E^{X_{i}^{k}}, E^{X_{i}^{2} X_{j}}$ and $E^{X_{i} X_{j}}$ for $n$ exponentially distributed risks linked by a multivariate FGM copula. Let $\vec{X}=$ $\left(X_{1}, X_{2}, \cdots, X_{n}\right), X_{1}, X_{2}, \cdots, X_{n}$ are $n$ exponentially distributed with $\lambda_{1}, \lambda_{2}, \cdots, \lambda_{n}$. As in the bivariate case, we assume that $\lambda_{i} \neq \lambda_{j}$ and $\lambda_{i} \neq 2 \lambda_{j}$ for $i \neq j$.

Proposition 4.2. Let $\vec{X}=\left(X_{1}, X_{2}, \cdots, X_{n}\right), X_{1}, X_{2}, \cdots, X_{n}$ are $n$ exponentially distributed with parameters $\lambda_{1}, \lambda_{2}, \cdots, \lambda_{n}$. The dependence structure for $\left(X_{1}, X_{2}, \cdots, X_{n}\right)$ is defined by a multivariate FGM copula. Then quantities $E^{X_{i}^{k}}, E^{X_{i}^{2} X_{j}}$ and $E^{X_{i} X_{j}}, i \neq j, i, j=1,2, \cdots, n, k=1,2,3$, are presented as follows:

$$
\begin{aligned}
& E^{X_{i}^{v} X_{j}}=\frac{(-1)^{n-2} \eta}{1-\kappa}\left\{\bar{L}_{X_{i}^{v} X_{j}}\left(\operatorname{VaR}_{\kappa}(S) ; \lambda_{1} ; \cdots ; \lambda_{n}\right)+\sum_{q=2}^{n} \sum_{1 \leq p_{1}<\cdots<p_{q} \leq n \bigcap i \cap j \in\left\{p_{1}, \cdots, p_{q}\right\}}\right. \\
& \theta_{p_{1} \cdots p_{q}}\left(\sum _ { l = 0 } ^ { q } \sum _ { ( \epsilon _ { 1 } , \cdots , \epsilon _ { q } ) - i - j \in \tau _ { l , q - 2 } } \left[(-1)^{l} 2^{q-2-l} \times\right.\right. \\
& \bar{L}_{X_{i}^{v} X_{j}}\left(\operatorname{VaR}_{\kappa}(S) ; 2^{\epsilon_{1}} \lambda_{p_{1}} ; \cdots ; 2^{\epsilon_{q}} \lambda_{p_{q}} ; \lambda_{i_{q+1}} ; \cdots ; \lambda_{i_{n}}\right) \\
& +(-1)^{l+1} 2^{q-2-l} \bar{M}_{X_{i}^{v} X_{j}}\left(\operatorname{VaR}_{\kappa}(S) ; 2^{\epsilon_{1}} \lambda_{p_{1}} ; \cdots ; 2^{\epsilon_{q}} \lambda_{p_{q}} ; \lambda_{i_{q+1}} ; \cdots ; \lambda_{i_{n}}\right) \\
& +(-1)^{l+1} 2^{q-2-l} \bar{N}_{X_{i}^{v} X_{j}}\left(\operatorname{VaR}_{\kappa}(S) ; 2^{\epsilon_{1}} \lambda_{p_{1}} ; \cdots ; 2^{\epsilon_{q}} \lambda_{p_{q}} ; \lambda_{i_{q+1}} ; \cdots ; \lambda_{i_{n}}\right) \\
& \left.\left.+(-1)^{l} 2^{q-2-l} \bar{Q}_{X_{i}^{v} X_{j}}\left(\operatorname{VaR}_{\kappa}(S) ; 2^{\epsilon} \lambda_{p_{1}} ; \cdots ; 2^{\epsilon} \lambda_{p_{q}} ; \lambda_{i_{q+1}} ; \cdots ; \lambda_{i_{n}}\right)\right]\right)
\end{aligned}
$$

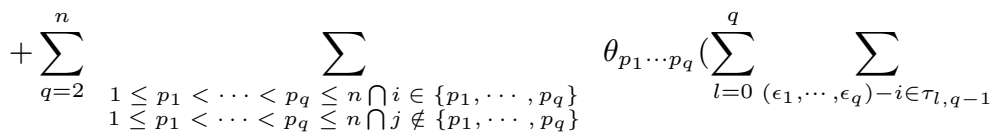

$$
\begin{aligned}
& {\left[(-1)^{l+1} 2^{q-1-l} \bar{L}_{X_{i}^{v} X_{j}}\left(\operatorname{VaR}_{\kappa}(S) ; 2^{\epsilon_{1}} \lambda_{p_{1}} ; \cdots ; 2^{\epsilon_{q}} \lambda_{p_{q}} ; \lambda_{i_{q+1}} ; \cdots ; \lambda_{i_{n}}\right)\right.} \\
& \left.\left.+(-1)^{l} 2^{q-1-l} \bar{M}_{X_{i}^{v} X_{j}}\left(\operatorname{VaR}_{\kappa}(S) ; 2^{\epsilon_{1}} \lambda_{p_{1}} ; \cdots ; 2^{\epsilon_{q}} \lambda_{p_{q}} ; \lambda_{i_{q+1}} ; \cdots ; \lambda_{i_{n}}\right)\right]\right)
\end{aligned}
$$

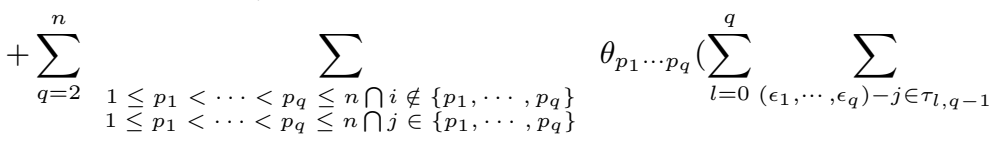

$$
\begin{aligned}
& {\left[(-1)^{l+1} 2^{q-1-l} \bar{L}_{X_{i}^{v} X_{j}}\left(\operatorname{VaR}_{\kappa}(S) ; 2^{\epsilon_{1}} \lambda_{p_{1}} ; \cdots ; 2^{\epsilon_{q}} \lambda_{p_{q}} ; \lambda_{i_{q+1}} ; \cdots ; \lambda_{i_{n}}\right)\right.}
\end{aligned}
$$




$$
\begin{aligned}
& \left.\left.+(-1)^{l} 2^{q-1-l} \bar{N}_{X_{i}^{v} X_{j}}\left(\operatorname{VaR}_{\kappa}(S) ; 2^{\epsilon_{1}} \lambda_{p_{1}} ; \cdots ; 2^{\epsilon_{q}} \lambda_{p_{q}} ; \lambda_{i_{q+1}} ; \cdots ; \lambda_{i_{n}}\right)\right]\right) \\
& +\sum_{q=2}^{n} \sum_{\substack{1 \leq p_{1}<\cdots<p_{q} \leq n \cap i \notin\left\{p_{1}, \cdots, p_{q}\right\} \\
1 \leq p_{1}<\cdots<p_{q} \leq n \cap j \notin\left\{p_{1}, \cdots, p_{q}\right\}}} \theta_{p_{1} \cdots p_{q}}\left(\sum_{l=0}^{q} \sum_{\left(\epsilon_{1}, \cdots, \epsilon_{q}\right) \in \tau_{l, q}}(-1)^{l} 2^{q-l}\right. \\
& \left.\left.\bar{L}_{X_{i}^{v} X_{j}}\left(\operatorname{VaR}_{\kappa}(S) ; 2^{\epsilon_{1}} \lambda_{p_{1}} ; \cdots ; 2^{\epsilon_{q}} \lambda_{p_{q}} ; \lambda_{i_{q+1}} ; \cdots ; \lambda_{i_{n}}\right)\right)\right\}, v=1,2, i \neq j \text {, } \\
& E^{X_{i}^{k}}=\frac{(-1)^{n-1} \eta}{1-\kappa}\left\{\bar{L}_{X_{i}^{k}}\left(\operatorname{VaR}_{\kappa}(S) ; \lambda_{1} ; \cdots ; \lambda_{n}\right)+\sum_{q=2}^{n} \sum_{1 \leq p_{1}<\cdots<p_{q} \leq n \cap i \in\left\{p_{1}, \cdots, p_{q}\right\}} \theta_{p_{1} \cdots p_{q}} \times\right. \\
& {\left[\sum _ { l = 0 } ^ { q - 1 } \sum _ { ( \epsilon _ { 1 } , \cdots , \epsilon _ { q } ) - i \in \tau _ { l , q - 1 } } \left\{(-1)^{l+1} 2^{q-1-l} \bar{L}_{X_{i}^{k}}\left(\operatorname{VaR}_{\kappa}(S) ; 2^{\epsilon_{1}} \lambda_{p_{1}} ; \cdots ; 2^{\epsilon_{q}} \lambda_{p_{q}} ; \lambda_{i_{q+1}} \cdots ; \lambda_{i_{n}}\right)\right.\right.} \\
& \left.\left.+(-1)^{l} 2^{q-1-l} \bar{M}_{X_{i}^{k}}\left(\operatorname{VaR}_{\kappa}(S) ; 2^{\epsilon_{1}} \lambda_{p_{1}} ; \cdots ; 2^{\epsilon_{q}} \lambda_{p_{q}} ; \lambda_{i_{q+1}} \cdots ; \lambda_{i_{n}}\right)\right\}\right] \\
& +\sum_{q=2}^{n} \sum_{1 \leq p_{1}<\cdots<p_{q} \leq n \cap i \notin\left\{p_{1}, \cdots, p_{q}\right\}} \theta_{p_{1} \cdots p_{q}}\left[\sum_{l=0}^{q} \sum_{\left(\epsilon_{1}, \cdots, \epsilon_{q}\right) \in \tau_{l, q}}(-1)^{l} 2^{q-l} \times\right. \\
& \left.\left.\bar{L}_{X_{i}^{k}}\left(\operatorname{VaR}_{\kappa}(S) ; 2^{\epsilon_{1}} \lambda_{p_{1}} ; \cdots ; 2^{\epsilon_{q}} \lambda_{p_{q}} ; \lambda_{i_{q+1}} \cdots ; \lambda_{i_{n}}\right)\right]\right\}, \quad k=1,2,3,
\end{aligned}
$$

where $\eta=\lambda_{1} \times \cdots \times \lambda_{n}, i_{q+1}, \cdots, i_{n}$ are the missing indexes of $p_{1}, \cdots, p_{q}$ to complete $1, \cdots, n, \tau_{l, q}$ are the sets of $q$-tuples composed of $l$ zeros and $q-l$ ones, for $l=0,1, \cdots, q$ and $q=2, \cdots, n, \bar{L}_{X_{i}^{k}}\left(x ; \alpha_{1} ; \cdots ; \alpha_{i-1} ; \alpha_{i+1} ; \cdots ; \alpha_{n}\right)$ and $\bar{M}_{X_{i}^{k}}\left(x ; \alpha_{1} ; \cdots ; \alpha_{i-1} ; \alpha_{i+1} ;\right.$ $\left.\cdots ; \alpha_{n}\right)$ are the $(n-2)$ th order divided differences of $\bar{L}_{X_{i}^{k}}(x ; \alpha)$ and $\bar{M}_{X_{i}^{k}}(x ; \alpha)$, respectively, and $\bar{L}_{X_{i}^{v} X_{j}}\left(x ; \alpha_{1} ; \cdots ; \alpha_{i-1} ; \alpha_{i+1} ; \cdots ; \alpha_{j-1} ; \alpha_{j+1} ; \cdots ; \alpha_{n}\right), \bar{M}_{X_{i}^{v} X_{j}}\left(x ; \alpha_{1} ; \cdots ; \alpha_{i-1}\right.$; $\left.\alpha_{i+1} ; \cdots ; \alpha_{j-1} ; \alpha_{j+1} ; \cdots ; \alpha_{n}\right), \bar{N}_{X_{i}^{v} X_{j}}\left(x ; \alpha_{1} ; \cdots ; \alpha_{i-1} ; \alpha_{i+1} ; \cdots ; \alpha_{j-1} ; \alpha_{j+1} ; \cdots ; \alpha_{n}\right)$, and $\bar{Q}_{X_{i}^{v} X_{j}}\left(x ; \alpha_{1} ; \cdots ; \alpha_{i-1} ; \alpha_{i+1} ; \cdots ; \alpha_{j-1} ; \alpha_{j+1} ; \cdots ; \alpha_{n}\right)$ are the $(n-3)$ th order divided differences of $\bar{L}_{X_{i}^{v} X_{j}}(x ; \alpha), \bar{M}_{X_{i}^{v} X_{j}}(x ; \alpha), \bar{N}_{X_{i}^{v} X_{j}}(x ; \alpha)$ and $\bar{Q}_{X_{i}^{v} X_{j}}(x ; \alpha)$, respectively. The expressions of $\bar{L}_{X_{i}^{k}}(x ; \alpha)$ and $\bar{M}_{X_{i}^{k}}(x ; \alpha)$ are given in Eqs. (4.27)-(4.32) and the expressions of $\bar{L}_{X_{i}^{v} X_{j}}(x ; \alpha), \bar{M}_{X_{i}^{v} X_{j}}(x ; \alpha), \bar{N}_{X_{i}^{v} X_{j}}(x ; \alpha)$ and $\bar{Q}_{X_{i}^{v} X_{j}}(x ; \alpha)$ are given in Eqs. (4.14)-(4.21).

Proof. We first calculate the explicit the formulae of $E^{X_{i}^{2} X_{j}}, i \neq j, i, j=1,2, \cdots, n$.

$$
\begin{gathered}
E^{X_{i}^{2} X_{j}}=\mathbf{E}\left[X_{i}^{2} X_{j} \mid S>\operatorname{VaR}_{\kappa}(S)\right]=\int_{\operatorname{VaR}_{\kappa}(S)}^{\infty} \mathbf{E}\left[X_{i}^{2} X_{j} \mid S=s\right] f_{S \mid S>\operatorname{VaR}_{\kappa}(S)}(s) \mathrm{d} s \\
=\frac{\int_{\operatorname{VaR}_{\kappa}(S)}^{\infty} \int_{0}^{s} \int_{0}^{s-x_{i}} x_{i}^{2} x_{j} f_{X_{i}, X_{j}, S}\left(x_{i}, x_{j}, s\right) \mathrm{d} x_{j} \mathrm{~d} x_{i} \mathrm{~d} s}{1-\kappa} .
\end{gathered}
$$

In order to evaluate Eq.(4.4), we need to derive a recursive formula for $f_{X_{i}, X_{j}, S}\left(x_{i}, x_{j}, s\right)=$ $f_{X_{i}, X_{j}, S-X_{i}-X_{j}}\left(x_{i}, x_{j}, s-x_{i}-x_{j}\right)$. Given that the random variables $X_{1}, X_{1}, \cdots, X_{n}$ are not independent, we cannot separate $f_{X_{i}, X_{j}, S-X_{i}-X_{j}}\left(x_{i}, x_{j}, s-x_{i}-x_{j}\right)$ into the product of $f_{X_{i}}\left(x_{i}\right), f_{X_{j}}\left(x_{j}\right)$ and $f_{S-X_{i}-X_{j}}\left(s-x_{i}-x_{j}\right)$. Now, we derive the recursive formula for $f_{X_{i}, X_{j}, S-X_{i}-X_{j}}\left(x_{i}, x_{j}, s-x_{i}-x_{j}\right)$. First, we rewrite the $f_{X_{1}, \ldots, X_{n}}\left(x_{1}, x_{2}, \cdots, x_{n}\right)$ defined in Eq. (2.2) as

$$
\begin{aligned}
& f_{X_{1}, \ldots, X_{n}}\left(x_{1}, \ldots, x_{n}\right)=f_{X_{i}}\left(x_{i}\right) f_{X_{j}}\left(x_{j}\right)\left\{\omega_{-i-j}\left(x_{1} ; \cdots ; x_{n} ; \lambda_{1} ; \cdots ; \lambda_{n}\right)\right. \\
& +\sum_{q=2}^{n} \sum_{\substack{1 \leq p_{1}<\ldots<p_{q} \leq n \\
i \cap j \in\left\{p_{1}, \cdots, p_{q}\right\}}} \theta_{p_{1} \cdots p_{q}}\left(1-2 F_{X_{i}}\left(x_{i}\right)\right)\left(1-2 F_{X_{j}}\left(x_{j}\right)\right)\left[\sum_{l=0}^{q} \sum_{\left(\epsilon_{1}, \cdots, \epsilon_{q}\right)-i-j \in \tau_{l, q-2}}\right. \\
& \left.(-1)^{l} \omega_{-i-j}\left(x_{p_{1}} ; \cdots ; x_{p_{q}} ; x_{i_{q+1}} ; \cdots ; x_{i_{n}} ; 2^{\epsilon_{1}} \lambda_{p_{1}} ; \cdots ; 2^{\epsilon_{q}} \lambda_{p_{q}} ; \lambda_{i_{q+1}} \cdots ; \lambda_{i_{n}}\right)\right]
\end{aligned}
$$




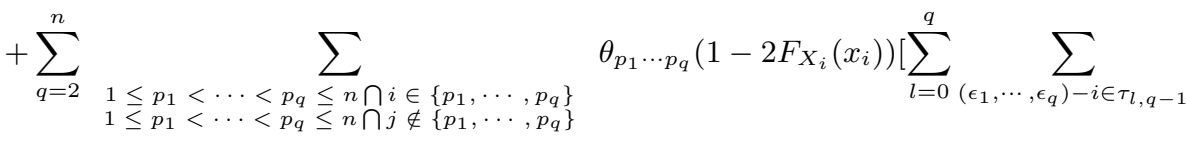

$$
\begin{aligned}
& \left.(-1)^{l} \omega_{-i-j}\left(x_{p_{1}} ; \cdots ; x_{p_{q}} ; x_{i_{q+1}} ; \cdots ; x_{i_{n}} ; 2^{\epsilon_{1}} \lambda_{p_{1}} ; \cdots ; 2^{\epsilon_{q}} \lambda_{p_{q}} ; \lambda_{i_{q+1}} \cdots ; \lambda_{i_{n}}\right)\right] \\
& +\sum_{q=2}^{n} \sum_{\substack{1 \leq p_{1}<\cdots<p_{q} \leq n \cap i \notin\left\{p_{1}, \cdots, p_{q}\right\} \\
1 \leq p_{1}<\cdots<p_{q} \leq n \cap j \in\left\{p_{1}, \cdots, p_{q}\right\}}} \theta_{p_{1} \cdots p_{q}}\left(1-2 F_{X_{j}}\left(x_{j}\right)\right)\left[\sum_{l=0}^{q} \sum_{\left(\epsilon_{1}, \cdots, \epsilon_{q}\right)-j \in \tau_{l, q-1}}\right. \\
& \left.(-1)^{l} \omega_{-i-j}\left(x_{p_{1}} ; \cdots ; x_{p_{q}} ; x_{i_{q+1}} ; \cdots ; x_{i_{n}} ; 2^{\epsilon_{1}} \lambda_{p_{1}} ; \cdots ; 2^{\epsilon_{q}} \lambda_{p_{q}} ; \lambda_{i_{q+1}} \cdots ; \lambda_{i_{n}}\right)\right] \\
& +\sum_{q=2}^{n} \sum_{\substack{1 \leq p_{1}<\cdots<p_{q} \leq n \cap i \notin\left\{p_{1}, \ldots, p_{q}\right\} \\
1 \leq p_{1}<\cdots<p_{q} \leq n \cap j \notin\left\{p_{1}, \cdots, p_{q}\right\}}} \theta_{p_{1} \cdots p_{q}}\left[\sum_{l=0}^{q} \sum_{\left(\epsilon_{1}, \cdots, \epsilon_{q}\right) \in \tau_{l, q}}\right. \\
& \left.\left.(-1)^{l} \omega_{-i-j}\left(x_{p_{1}} ; \cdots ; x_{p_{q}} ; x_{i_{q+1}} ; \cdots ; x_{i_{n}} ; 2^{\epsilon_{1}} \lambda_{p_{1}} ; \cdots ; 2^{\epsilon_{q}} \lambda_{p_{q}} ; \lambda_{i_{q+1}} \cdots ; \lambda_{i_{n}}\right)\right]\right\},
\end{aligned}
$$

where $\omega_{-i-j}\left(x_{1} ; x_{2} ; \cdots ; x_{n} ; \alpha_{1} ; \alpha_{2} ; \cdots ; \alpha_{n}\right)=\alpha_{1} e^{-\alpha_{1} x_{1}} \times \cdots \times \alpha_{i-1} e^{-\alpha_{i-1} x_{i-1}} \times \alpha_{i+1}$ $e^{-\alpha_{i+1} x_{i+1}} \times \cdots \times \alpha_{j-1} e^{-\alpha_{j-1} x_{j-1}} \times \alpha_{j+1} e^{-\alpha_{j+1} x_{j+1}} \cdots \alpha_{n} e^{-\alpha_{n} x_{n}}$. Assume that

$$
\begin{gathered}
\int_{0}^{s-x_{i}-x_{j}} \int_{0}^{s-x_{1}-x_{i}-x_{j}} \ldots \int_{0}^{s-x_{1}-\cdots-x_{n-1}} \omega_{-i-j}\left(x_{1} ; \ldots ; x_{n} ; \alpha_{1} ; \ldots ; \alpha_{n}\right) \mathrm{d} x_{1} \cdots \mathrm{d} x_{i-1} \times \\
=\sum_{l=1, l \neq i, l \neq j}^{n}\left(\prod_{q=1, q \neq l, q \neq i, q \neq j} \frac{\alpha_{q}}{\alpha_{q}-\alpha_{l}}\right) \alpha_{l} e^{-\alpha_{l}\left(s-x_{i}-x_{j}\right)}=h_{-i-j}\left(s-x_{i}-x_{j} ; \alpha_{1} ; \ldots ; \alpha_{n}\right) .
\end{gathered}
$$

Note that

$$
\begin{aligned}
& f_{X_{i}, X_{j}, S-X_{i}-X_{j}}\left(x_{i}, x_{j}, s-x_{i}-x_{j}\right) \\
&= f_{X_{i}, X_{j}, X_{1}+\cdots+X_{i-1}+X_{i+1}+\cdots+X_{j-1}+X_{j+1}+\cdots+X_{n}}\left(x_{i}, x_{j}, s-x_{i}-x_{j}\right) \\
&= \int_{0}^{s-x_{i}-x_{j}} \int_{0}^{s-x_{1}-x_{i}-x_{j}} \cdots \int_{0}^{s-x_{1}-\cdots-x_{n-1}} f_{X_{1}, \ldots, X_{n}}\left(x_{1}, \ldots, x_{n-1}, s-x_{1}-\cdots-x_{n-1}\right) \\
& \times \mathrm{d} x_{1} \cdots \mathrm{d} x_{i-1} \mathrm{~d} x_{i+1} \cdots \mathrm{d} x_{j-1} \mathrm{~d} x_{j+1} \cdots \mathrm{d} x_{n-1} .
\end{aligned}
$$

Plugging (4.5) into (4.7), we have

$$
\begin{aligned}
& f_{X_{i}, X_{j}, S-X_{i}-X_{j}}\left(x_{i}, x_{j}, s-x_{i}-x_{j}\right) \\
& =f_{X_{i}}\left(x_{i}\right) f_{X_{j}}\left(x_{j}\right)\left\{h_{-i-j}\left(s-x_{i}-x_{j} ; \lambda_{1} ; \cdots ; \lambda_{i-1} ; \lambda_{i+1} ; \cdots ; \lambda_{j-1} ; \cdots ; \lambda_{j+1} ; \cdots ; \lambda_{n}\right)\right. \\
& +\sum_{q=2}^{n} \sum_{1 \leq p_{1}<\cdots<p_{q} \leq n \cap i \cap j \in\left\{p_{1}, \cdots, p_{q}\right\}} \theta_{p_{1} \cdots p_{q}}\left(1-2 F_{X_{i}}\left(x_{i}\right)\right)\left(1-2 F_{X_{j}}\left(x_{j}\right)\right) \times \\
& {\left[\sum_{l=0}^{q} \sum_{\left(\epsilon_{1}, \cdots, \epsilon_{q}\right)-i-j \in \tau_{l, q-2}}(-1)^{l} h_{-i-j}\left(s-x_{i}-x_{j} ; 2^{\epsilon_{1}} \lambda_{p_{1}} ; \cdots ; 2^{\epsilon_{q}} \lambda_{p_{q}} ; \lambda_{i_{q+1}} \cdots ; \lambda_{i_{n}}\right)\right]} \\
& +\sum_{q=2}^{n} \sum_{\substack{1 \leq p_{1}<\cdots<p_{q} \leq n \cap i \in\left\{p_{1}, \cdots, p_{q}\right\} \\
1 \leq p_{1}<\cdots<p_{q} \leq n \cap j \notin\left\{p_{1}, \cdots, p_{q}\right\}}} \theta_{p_{1} \cdots p_{q}}\left(1-2 F_{X_{i}}\left(x_{i}\right)\right) \times \\
& {\left[\sum_{l=0}^{q} \sum_{\left(\epsilon_{1}, \cdots, \epsilon_{q}\right)-i \in \tau_{l, q-1}}(-1)^{l} h_{-i-j}\left(s-x_{i}-x_{j} ; 2^{\epsilon_{1}} \lambda_{p_{1}} ; \cdots ; 2^{\epsilon_{q}} \lambda_{p_{q}} ; \lambda_{i_{q}+1} \cdots ; \lambda_{i_{n}}\right)\right]}
\end{aligned}
$$




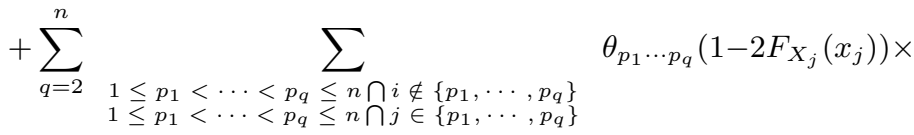

$$
\begin{aligned}
& {\left[\sum_{l=0}^{q} \sum_{\left(\epsilon_{1}, \cdots, \epsilon_{q}\right)-j \in \tau_{l, q-1}}(-1)^{l} h_{-i-j}\left(s-x_{i}-x_{j} ; 2^{\epsilon_{1}} \lambda_{p_{1}} ; \cdots ; 2^{\epsilon_{q}} \lambda_{p_{q}} ; \lambda_{i_{q+1}} \cdots ; \lambda_{i_{n}}\right)\right]} \\
& +\sum_{q=2}^{n} \sum_{\substack{1 \leq p_{1}<\cdots<p_{q} \leq n \cap i \notin\left\{p_{1}, \cdots, p_{q}\right\} \\
1 \leq p_{1}<\cdots<p_{q} \leq n \cap j \notin\left\{p_{1}, \cdots, p_{q}\right\}}} \theta_{p_{1} \cdots p_{q}} \times \\
& {\left[\sum_{l=0}^{q} \sum_{\left(\epsilon_{1}, \cdots, \epsilon_{q}\right) \in \tau_{l, q}}(-1)^{l} h_{-i-j}\left(s-x_{i}-x_{j} ; 2^{\epsilon_{1}} \lambda_{p_{1}} ; \cdots ; 2^{\epsilon_{q}} \lambda_{p_{q}} ; \lambda_{i_{q+1}} \cdots ; \lambda_{i_{n}}\right)\right] .}
\end{aligned}
$$

Utilizing the dividend difference presented in Chiragiev and Landsman [6], note that

$$
h_{-i-j}\left(x ; \alpha_{1} ; \cdots ; \alpha_{i-1} ; \alpha_{i+1} ; \cdots ; \alpha_{j-1} ; \alpha_{j+1} ; \cdots ; \alpha_{n}\right)=(-1)^{n-3} \times \alpha_{1} \times \cdots \times \alpha_{i-1} \times
$$

$\alpha_{i+1} \times \cdots \times \alpha_{j-1} \times \alpha_{j+1} \times \cdots \times \alpha_{n} \times \bar{\Lambda}\left(x ; \alpha_{1} ; \cdots ; \alpha_{i-1} ; \alpha_{i+1} ; \cdots ; \alpha_{j-1} ; \alpha_{j+1} ; \cdots ; \alpha_{n}\right)$, where $\bar{\Lambda}\left(x ; \alpha_{1} ; \cdots ; \alpha_{i-1} ; \alpha_{i+1} ; \cdots ; \alpha_{j-1} ; \alpha_{j+1} ; \cdots ; \alpha_{n}\right)$ is the $(n-3)$ th order divided difference of $\bar{\Lambda}(x ; \alpha)=e^{-\alpha x}$. Then, we get

$$
\begin{aligned}
& \int_{0}^{s} \int_{0}^{s-x_{i}} x_{i}^{2} x_{j} f_{X_{i}}\left(x_{i}\right) f_{X_{j}}\left(x_{j}\right) h_{-i-j}\left(s-x_{i}-x_{j} ; \lambda_{1} ; \cdots ; \lambda_{i-1} ;\right. \\
&\left.\lambda_{i+1} ; \cdots ; \lambda_{j-1} ; \cdots ; \lambda_{j+1} ; \cdots ; \lambda_{n}\right) \mathrm{d} x_{j} \mathrm{~d} x_{i} \\
&=\int_{0}^{s} \int_{0}^{s-x_{i}} x_{i}^{2} x_{j} f_{X_{i}}\left(x_{i}\right) f_{X_{j}}\left(x_{j}\right)(-1)^{n-3} \eta_{-i-j} \bar{\Lambda}\left(x ; \lambda_{1} ; \cdots ; \lambda_{i-1} ;\right. \\
&\left.\lambda_{i+1} ; \cdots ; \lambda_{j-1} ; \lambda_{j+1} ; \cdots ; \lambda_{n}\right) \mathrm{d} x_{j} \mathrm{~d} x_{i} \\
&=(-1)^{n-2} \eta L_{X_{i}^{2} X_{j}}\left(s ; \lambda_{1} ; \cdots ; \lambda_{i-1} ; \lambda_{i+1} ; \cdots ; \lambda_{j-1} ; \lambda_{j+1} ; \cdots ; \lambda_{n}\right),
\end{aligned}
$$

where $\eta_{-i-j}=\lambda_{1} \times \cdots \times \lambda_{i-1} \times \lambda_{i+1} \times \cdots \times \lambda_{j-1} \times \lambda_{j+1} \times \cdots \times \lambda_{n}, L_{X_{i}^{2} X_{j}}(s ; \lambda)=$ $-\int_{0}^{s} \int_{0}^{s-x_{i}} x_{i}^{2} x_{j} e^{-\lambda_{i} x_{i}} e^{-\lambda_{j} x_{j}} \bar{\Lambda}\left(s-x_{i}-x_{j} ; \lambda\right) \mathrm{d} x_{j} \mathrm{~d} x_{i}$. Let also $M_{X_{i}^{2} X_{j}}(s ; \lambda)=-\int_{0}^{s} \int_{0}^{s-x_{i}}$ $2 x_{i}^{2} x_{j} e^{-2 \lambda_{i} x_{i}} e^{-\lambda_{j} x_{j}} \bar{\Lambda}\left(s-x_{i}-x_{j} ; \lambda\right) \mathrm{d} x_{j} \mathrm{~d} x_{i}, N_{X_{i}^{2} X_{j}}(s ; \lambda)=-\int_{0}^{s} \int_{0}^{s-x_{i}} 2 x_{i}^{2} x_{j} e^{-\lambda_{i} x_{i}} e^{-2 \lambda_{j} x_{j}}$ $\bar{\Lambda}\left(s-x_{i}-x_{j} ; \lambda\right) \mathrm{d} x_{j} \mathrm{~d} x_{i}$, and $O_{X_{i}^{2} X_{j}}(s ; \lambda)=-\int_{0}^{s} \int_{0}^{s-x_{i}} 4 x_{i}^{2} x_{j} e^{-2 \lambda_{i} x_{i}} e^{-2 \lambda_{j} x_{j}} \bar{\Lambda}\left(s-x_{i}-\right.$ $\left.x_{j} ; \lambda\right) \mathrm{d} x_{j} \mathrm{~d} x_{i}, i \neq j, i, j=1,2, \cdots, n$.

Given that the risks are exponentially distributed, then $L_{X_{i}^{2} X_{j}}, M_{X_{i}^{2} X_{j}}, N_{X_{i}^{2} X_{j}}$ and $O_{X_{i}^{2} X_{j}}$ can be rewritten as

$$
\begin{gathered}
L_{X_{i}^{2} X_{j}}(s ; \lambda)=\frac{-2 e^{-\lambda s}}{\left(\lambda_{i}-\lambda\right)^{3}\left(\lambda_{j}-\lambda\right)^{2}}+\frac{2\left(\lambda_{i}-4 \lambda_{j}+3 \lambda\right) e^{-\lambda_{j} s}}{\left(\lambda_{i}-\lambda_{j}\right)^{4}\left(\lambda_{j}-\lambda\right)^{2}}+\frac{\left.2 s\left(3 \lambda_{i}-\lambda_{j}-2 \lambda\right)\right) e^{-\lambda_{i} s}}{\left(\lambda_{i}-\lambda_{j}\right)^{3}\left(\lambda_{i}-\lambda\right)^{2}} \\
+\frac{2 s e^{-\lambda_{j} s}}{\left(\lambda_{i}-\lambda_{j}\right)^{3}\left(\lambda_{j}-\lambda\right)}+\frac{s^{2} e^{-\lambda_{i} s}}{\left(\lambda_{i}-\lambda_{j}\right)^{2}\left(\lambda_{i}-\lambda\right)}+\frac{2\left(6 \lambda_{i}^{2}+\lambda_{j}^{2}+2 \lambda \lambda_{j}+3 \lambda^{2}-4 \lambda_{i}\left(\lambda_{j}+2 \lambda\right)\right) e^{-\lambda_{i} s}}{\left(\lambda_{i}-\lambda_{j}\right)^{4}\left(\lambda_{i}-\lambda\right)^{3}} \\
\begin{aligned}
& M_{X_{i}^{2} X_{j}}(s ; \lambda)=-2\left(\frac{2 e^{-\lambda s}}{\left(2 \lambda_{i}-\lambda\right)^{3}\left(\lambda_{j}-\lambda\right)^{2}}-\frac{2\left(2 \lambda_{i}-4 \lambda_{j}+3 \lambda\right) e^{-\lambda_{j} s}}{\left(2 \lambda_{i}-\lambda_{j}\right)^{4}\left(\lambda_{j}-\lambda\right)^{2}}-\frac{2 s e^{-\lambda_{j} s}}{\left(2 \lambda_{i}-\lambda_{j}\right)^{3}\left(\lambda_{j}-\lambda\right)}\right. \\
&-\frac{s^{2} e^{-2 \lambda_{i} s}}{\left(2 \lambda_{i}-\lambda_{j}\right)^{2}\left(2 \lambda_{i}-\lambda\right)}-\frac{\left.2 s\left(3\left(2 \lambda_{i}\right)-\lambda_{j}-2 \lambda\right)\right) e^{-2 \lambda_{i} s}}{\left(2 \lambda_{i}-\lambda_{j}\right)^{3}\left(2 \lambda_{i}-\lambda\right)^{2}} \\
&\left.-\frac{2\left(6\left(2 \lambda_{i}\right)^{2}+\lambda_{j}^{2}+2 \lambda \lambda_{j}+3 \lambda^{2}-4\left(2 \lambda_{i}\right)\left(\lambda_{j}+2 \lambda\right)\right) e^{-2 \lambda_{i} s}}{\left(2 \lambda_{i}-\lambda_{j}\right)^{4}\left(2 \lambda_{i}-\lambda\right)^{3}}\right), \\
& N_{X_{i}^{2} X_{j}}(s ; \lambda)=-2\left(\frac{2 e^{-\lambda s}}{\left(\lambda_{i}-\lambda\right)^{3}\left(2 \lambda_{j}-\lambda\right)^{2}}-\frac{2\left(\lambda_{i}-4\left(2 \lambda_{j}\right)+3 \lambda\right) e^{-2 \lambda_{j} s}}{\left(\lambda_{i}-2 \lambda_{j}\right)^{4}\left(2 \lambda_{j}-\lambda\right)^{2}}\right.
\end{aligned}
\end{gathered}
$$




$$
\begin{gathered}
-\frac{2 s e^{-2 \lambda_{j} s}}{\left(\lambda_{i}-2 \lambda_{j}\right)^{3}\left(2 \lambda_{j}-\lambda\right)}-\frac{s^{2} e^{-\lambda_{i} s}}{\left(\lambda_{i}-2 \lambda_{j}\right)^{2}\left(\lambda_{i}-\lambda\right)}-\frac{\left.2 s\left(3 \lambda_{i}-2 \lambda_{j}-2 \lambda\right)\right) e^{-\lambda_{i} s}}{\left(\lambda_{i}-2 \lambda_{j}\right)^{3}\left(\lambda_{i}-\lambda\right)^{2}} \\
\left.-\frac{2\left(6 \lambda_{i}^{2}+\left(2 \lambda_{j}\right)^{2}+2 \lambda 2 \lambda_{j}+3 \lambda^{2}-4 \lambda_{i}\left(2 \lambda_{j}+2 \lambda\right)\right) e^{-\lambda_{i} s}}{\left(\lambda_{i}-2 \lambda_{j}\right)^{4}\left(\lambda_{i}-\lambda\right)^{3}}\right), \\
O_{X_{i}^{2} X_{j}}(s ; \lambda)=-4\left(\frac{2 e^{-\lambda s}}{\left(2 \lambda_{i}-\lambda\right)^{3}\left(2 \lambda_{j}-\lambda\right)^{2}}-\frac{2\left(2 \lambda_{i}-4\left(2 \lambda_{j}\right)+3 \lambda\right) e^{-2 \lambda_{j} s}}{\left(2 \lambda_{i}-2 \lambda_{j}\right)^{4}\left(2 \lambda_{j}-\lambda\right)^{2}}\right. \\
-\frac{2 s e^{-2 \lambda_{j} s}}{\left(2 \lambda_{i}-2 \lambda_{j}\right)^{3}\left(2 \lambda_{j}-\lambda\right)}-\frac{s^{2} e^{-2 \lambda_{i} s}}{\left(2 \lambda_{i}-2 \lambda_{j}\right)^{2}\left(2 \lambda_{i}-\lambda\right)}-\frac{\left.2 s\left(3\left(2 \lambda_{i}\right)-2 \lambda_{j}-2 \lambda\right)\right) e^{-2 \lambda_{i} s}}{\left(2 \lambda_{i}-2 \lambda_{j}\right)^{3}\left(2 \lambda_{i}-\lambda\right)^{2}} \\
\left.-\frac{2\left(6\left(2 \lambda_{i}\right)^{2}+\left(2 \lambda_{j}\right)^{2}+2 \lambda 2 \lambda_{j}+3 \lambda^{2}-4\left(2 \lambda_{i}\right)\left(2 \lambda_{j}+2 \lambda\right)\right) e^{-2 \lambda_{i} s}}{\left(2 \lambda_{i}-2 \lambda_{j}\right)^{4}\left(2 \lambda_{i}-\lambda\right)^{3}}\right) .
\end{gathered}
$$

Utilizing the results of (4.8) and (4.10)-(4.13), one can rewrite $\int_{0}^{s} \int_{0}^{s-x_{i}} x_{i}^{2} x_{j} f_{x_{i}, x_{j}, S} \mathrm{~d} x_{j} \mathrm{~d} x_{i}$ as

$$
\begin{aligned}
& \int_{0}^{s} \int_{0}^{s-x_{i}} x_{i}^{2} x_{j} f_{x_{i}, x_{j}, S} \mathrm{~d} x_{j} \mathrm{~d} x_{i}=(-1)^{n-2} \eta\left\{L_{X_{i}^{2} X_{j}}\left(s ; \lambda_{1} ; \cdots ; \lambda_{n}\right)\right. \\
& +\sum_{q=2}^{n} \sum_{\substack{1 \leq p_{1}<\cdots<p_{q} \leq n \\
i \bigcap j \in\left\{p_{1}, \cdots, p_{q}\right\}}} \theta_{p_{1} \cdots p_{q}}\left(\sum_{l=0}^{q} \sum_{\left(\epsilon_{1}, \cdots, \epsilon_{q}\right)-i-j \in \tau_{l, q-2}}(-1)^{l} 2^{q-2-l} \times\right. \\
& {\left[L_{X_{i}^{2} X_{j}}\left(s ; 2^{\epsilon_{1}} \lambda_{p_{1}} ; \cdots ; 2^{\epsilon_{q}} \lambda_{p_{q}} ; \lambda_{i_{q+1}} ; \cdots ; \lambda_{i_{n}}\right)\right.} \\
& -M_{X_{i}^{2} X_{j}}\left(s ; 2^{\epsilon_{1}} \lambda_{p_{1}} ; \cdots ; 2^{\epsilon_{q}} \lambda_{p_{q}} ; \lambda_{i_{q+1}} ; \cdots ; \lambda_{i_{n}}\right) \\
& -N_{X_{i}^{2} X_{j}}\left(s ; 2^{\epsilon_{1}} \lambda_{p_{1}} ; \cdots ; 2^{\epsilon_{q}} \lambda_{p_{q}} ; \lambda_{i_{q+1}} ; \cdots ; \lambda_{i_{n}}\right) \\
& \left.\left.+Q_{X_{i}^{2} X_{j}}\left(s ; 2^{\epsilon_{1}} \lambda_{p_{1}} ; \cdots ; 2^{\epsilon_{q}} \lambda_{p_{q}} ; \lambda_{i_{q+1}} ; \cdots ; \lambda_{i_{n}}\right)\right]\right) \\
& -\sum_{q=2}^{n} \sum_{\substack{1 \leq p_{1}<\cdots<p_{q} \leq n \bigcap i \in\left\{p_{1}, \cdots, p_{q}\right\} \\
1 \leq p_{1}<\cdots<p_{q} \leq n \bigcap j \notin\left\{p_{1}, \cdots, p_{q}\right\}}} \theta_{p_{1} \cdots p_{q}}\left(\sum_{l=0}^{q} \sum_{\left(\epsilon_{1}, \cdots, \epsilon_{q}\right)-i \in \tau_{l, q-1}}(-1)^{l} 2^{q-1-l} \times\right. \\
& {\left[L_{X_{i}^{2} X_{j}}\left(s ; 2^{\epsilon_{1}} \lambda_{p_{1}} ; \cdots ; 2^{\epsilon_{q}} \lambda_{p_{q}} ; \lambda_{i_{q+1}} ; \cdots ; \lambda_{i_{n}}\right)\right.} \\
& \left.\left.-M_{X_{i}^{2} X_{j}}\left(s ; 2^{\epsilon_{1}} \lambda_{p_{1}} ; \cdots ; 2^{\epsilon q} \lambda_{p_{q}} ; \lambda_{i_{q+1}} ; \cdots ; \lambda_{i_{n}}\right)\right]\right)
\end{aligned}
$$

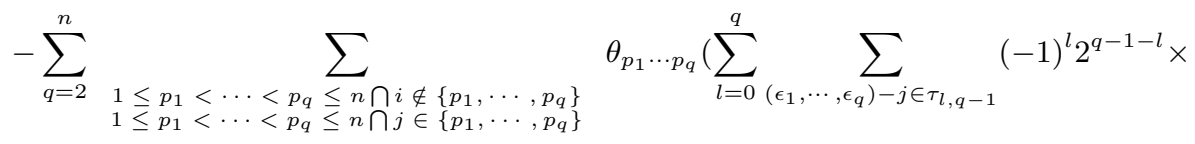

$$
\begin{aligned}
& {\left[L_{X_{i}^{2} X_{j}}\left(s ; 2^{\epsilon_{1}} \lambda_{p_{1}} ; \cdots ; 2^{\epsilon_{q}} \lambda_{p_{q}} ; \lambda_{i_{q+1}} ; \cdots ; \lambda_{i_{n}}\right)\right.} \\
& \left.\left.-N_{X_{i}^{2} X_{j}}\left(s ; 2^{\epsilon} \lambda_{p_{1}} ; \cdots ; 2^{\epsilon q} \lambda_{p_{q}} ; \lambda_{i_{q+1}} ; \cdots ; \lambda_{i_{n}}\right)\right]\right) \\
& +\sum_{q=2}^{n} \sum_{\substack{1 \leq p_{1}<\cdots<p_{q} \leq n \bigcap i \notin\left\{p_{1}, \cdots, p_{q}\right\} \\
1 \leq p_{1}<\cdots<p_{q} \leq n \bigcap j \notin\left\{p_{1}, \cdots, p_{q}\right\}}} \theta_{p_{1} \cdots p_{q}} \times \\
& \left(\sum_{l=0}^{q} \sum_{\left(\epsilon_{1}, \cdots, \epsilon_{q}\right) \in \tau_{l, q}}(-1)^{l} 2^{q-l} L_{X_{i}^{2} X_{j}}\left(s ; 2^{\epsilon_{1}} \lambda_{p_{1}} ; \cdots ; 2^{\epsilon_{q}} \lambda_{p_{q}} ; \lambda_{i_{q+1}} ; \cdots ; \lambda_{i_{n}}\right)\right) \text {. }
\end{aligned}
$$

In order to calculate the explicit the formulae of $E^{X_{i}^{2} X_{j}}, i \neq j$, as in (4.4), the $L_{X_{i}^{2} X_{j}}(s ; \lambda), M_{X_{i}^{2} X_{j}}(s ; \lambda), N_{X_{i}^{2} X_{j}}(s ; \lambda)$ and $O_{X_{i}^{2} X_{j}}(s ; \lambda)$ terms in the equation above must be integrated on $s$ as follows.

$$
\bar{L}_{X_{i}^{2} X_{j}}(V ; \lambda)=\int_{V}^{\infty} L_{X_{i}^{2} X_{j}}(s ; \lambda) \mathrm{d} s=\frac{2\left(\lambda_{i}-4 \lambda_{j}+3 \lambda\right) e^{-\lambda_{j} V}}{\lambda_{j}\left(\lambda_{i}-\lambda_{j}\right)^{4}\left(\lambda_{j}-\lambda\right)^{2}}+\frac{2\left(1+V \lambda_{j}\right) e^{-\lambda_{j} V}}{\lambda_{j}^{2}\left(\lambda_{i}-\lambda_{j}\right)^{3}\left(\lambda_{j}-\lambda\right)}
$$




$$
\begin{aligned}
& +\frac{\left(2+\lambda_{i} V\left(2+\lambda_{i} V\right)\right) e^{-\lambda_{i} V}}{\lambda_{i}^{3}\left(\lambda_{i}-\lambda_{j}\right)^{2}\left(\lambda_{i}-\lambda\right)}+\frac{\left.2\left(1+V \lambda_{i}\right)\left(3 \lambda_{i}-\lambda_{j}-2 \lambda\right)\right) e^{-\lambda_{i} V}}{\lambda_{i}^{2}\left(\lambda_{i}-\lambda_{j}\right)^{3}\left(\lambda_{i}-\lambda\right)^{2}} \\
& +\frac{2\left(6 \lambda_{i}^{2}+\lambda_{j}^{2}+2 \lambda \lambda_{j}+3 \lambda^{2}-4 \lambda_{i}\left(\lambda_{j}+2 \lambda\right)\right) e^{-\lambda_{i} V}}{\lambda_{i}\left(\lambda_{i}-\lambda_{j}\right)^{4}\left(\lambda_{i}-\lambda\right)^{3}}-\frac{2 e^{-\lambda V}}{\lambda\left(\lambda_{i}-\lambda\right)^{3}\left(\lambda_{j}-\lambda\right)^{2}}, \\
& \bar{M}_{X_{i}^{2} X_{j}}(V ; \lambda)=2\left(\frac{2\left(2 \lambda_{i}-4 \lambda_{j}+3 \lambda\right) e^{-\lambda_{j} V}}{\lambda_{j}\left(2 \lambda_{i}-\lambda_{j}\right)^{4}\left(\lambda_{j}-\lambda\right)^{2}}+\frac{2\left(1+V \lambda_{j}\right) e^{-\lambda_{j} V}}{\lambda_{j}^{2}\left(2 \lambda_{i}-\lambda_{j}\right)^{3}\left(\lambda_{j}-\lambda\right)}\right. \\
& +\frac{\left(2+2 \lambda_{i} V\left(2+2 \lambda_{i} V\right)\right) e^{-2 \lambda_{i} V}}{\left(2 \lambda_{i}\right)^{3}\left(2 \lambda_{i}-\lambda_{j}\right)^{2}\left(2 \lambda_{i}-\lambda\right)}+\frac{\left.2\left(1+V 2 \lambda_{i}\right)\left(3\left(2 \lambda_{i}\right)-\lambda_{j}-2 \lambda\right)\right) e^{-2 \lambda_{i} V}}{\left(2 \lambda_{i}\right)^{2}\left(2 \lambda_{i}-\lambda_{j}\right)^{3}\left(2 \lambda_{i}-\lambda\right)^{2}} \\
& \left.+\frac{2\left(6\left(2 \lambda_{i}\right)^{2}+\lambda_{j}^{2}+2 \lambda \lambda_{j}+3 \lambda^{2}-4\left(2 \lambda_{i}\right)\left(\lambda_{j}+2 \lambda\right)\right) e^{-2 \lambda_{i} V}}{2 \lambda_{i}\left(2 \lambda_{i}-\lambda_{j}\right)^{4}\left(2 \lambda_{i}-\lambda\right)^{3}}-\frac{2 e^{-\lambda V}}{\lambda\left(2 \lambda_{i}-\lambda\right)^{3}\left(\lambda_{j}-\lambda\right)^{2}}\right), \\
& \bar{N}_{X_{i}^{2} X_{j}}(V ; \lambda)=2\left(\frac{2\left(\lambda_{i}-4\left(2 \lambda_{j}\right)+3 \lambda\right) e^{-2 \lambda_{j} V}}{2 \lambda_{j}\left(\lambda_{i}-2 \lambda_{j}\right)^{4}\left(2 \lambda_{j}-\lambda\right)^{2}}+\frac{2\left(1+V 2 \lambda_{j}\right) e^{-2 \lambda_{j} V}}{\left(2 \lambda_{j}\right)^{2}\left(\lambda_{i}-2 \lambda_{j}\right)^{3}\left(2 \lambda_{j}-\lambda\right)}\right. \\
& +\frac{\left(2+\lambda_{i} V\left(2+\lambda_{i} V\right)\right) e^{-\lambda_{i} V}}{\lambda_{i}^{3}\left(\lambda_{i}-2 \lambda_{j}\right)^{2}\left(\lambda_{i}-\lambda\right)}+\frac{\left.2\left(1+V \lambda_{i}\right)\left(3 \lambda_{i}-2 \lambda_{j}-2 \lambda\right)\right) e^{-\lambda_{i} V}}{\lambda_{i}^{2}\left(\lambda_{i}-2 \lambda_{j}\right)^{3}\left(\lambda_{i}-\lambda\right)^{2}} \\
& \left.+\frac{2\left(6 \lambda_{i}^{2}+\left(2 \lambda_{j}\right)^{2}+2 \lambda 2 \lambda_{j}+3 \lambda^{2}-4 \lambda_{i}\left(2 \lambda_{j}+2 \lambda\right)\right) e^{-\lambda_{i} V}}{\lambda_{i}\left(\lambda_{i}-2 \lambda_{j}\right)^{4}\left(\lambda_{i}-\lambda\right)^{3}}-\frac{2 e^{-\lambda V}}{\lambda\left(\lambda_{i}-\lambda\right)^{3}\left(2 \lambda_{j}-\lambda\right)^{2}}\right), \\
& \bar{O}_{X_{i}^{2} X_{j}}(s ; \lambda)=4\left(\frac{2\left(2 \lambda_{i}-4\left(2 \lambda_{j}\right)+3 \lambda\right) e^{-2 \lambda_{j} V}}{2 \lambda_{j}\left(2 \lambda_{i}-2 \lambda_{j}\right)^{4}\left(2 \lambda_{j}-\lambda\right)^{2}}+\frac{2\left(1+V 2 \lambda_{j}\right) e^{-2 \lambda_{j} V}}{\left(2 \lambda_{j}\right)^{2}\left(2 \lambda_{i}-2 \lambda_{j}\right)^{3}\left(2 \lambda_{j}-\lambda\right)}\right. \\
& +\frac{\left(2+2 \lambda_{i} V\left(2+2 \lambda_{i} V\right)\right) e^{-2 \lambda_{i} V}}{\left(2 \lambda_{i}\right)^{3}\left(2 \lambda_{i}-2 \lambda_{j}\right)^{2}\left(2 \lambda_{i}-\lambda\right)}+\frac{\left.2\left(1+V 2 \lambda_{i}\right)\left(3\left(2 \lambda_{i}\right)-2 \lambda_{j}-2 \lambda\right)\right) e^{-2 \lambda_{i} V}}{\left(2 \lambda_{i}\right)^{2}\left(2 \lambda_{i}-2 \lambda_{j}\right)^{3}\left(2 \lambda_{i}-\lambda\right)^{2}} \\
& \left.+\frac{2\left(6\left(2 \lambda_{i}\right)^{2}+\left(2 \lambda_{j}\right)^{2}+2 \lambda 2 \lambda_{j}+3 \lambda^{2}-4\left(2 \lambda_{i}\right)\left(2 \lambda_{j}+2 \lambda\right)\right) e^{-2 \lambda_{i} V}}{2 \lambda_{i}\left(2 \lambda_{i}-2 \lambda_{j}\right)^{4}\left(2 \lambda_{i}-\lambda\right)^{3}}-\frac{2 e^{-\lambda V}}{\lambda\left(2 \lambda_{i}-\lambda\right)^{3}\left(2 \lambda_{j}-\lambda\right)^{2}}\right) .
\end{aligned}
$$

Finally, we can derive the explicit the formulae for $E^{X_{i}^{2} X_{j}}, i \neq j, i, j=1,2, \cdots, n$.

Since the formula of $E^{X_{i} X_{j}}$ can be calculated similarly, we omit the proof. We give the expressions of $\bar{L}_{X_{i} X_{j}}(V ; \lambda), \bar{M}_{X_{i} X_{j}}(V ; \lambda), \bar{N}_{X_{i} X_{j}}(V ; \lambda)$ and $\bar{O}_{X_{i} X_{j}}(V ; \lambda)$ directly,

$$
\begin{aligned}
& \bar{L}_{X_{i} X_{j}}(V ; \lambda)=-\left(\frac{e^{-\lambda V}}{\lambda\left(\lambda_{i}-\lambda\right)^{2}\left(\lambda_{j}-\lambda\right)^{2}}-\frac{\left(\lambda_{i}-3 \lambda_{j}+2 \lambda\right) e^{-\lambda_{j} V}}{\lambda_{j}\left(\lambda_{i}-\lambda_{j}\right)^{3}\left(\lambda_{j}-\lambda\right)^{2}}-\frac{\left(1+V \lambda_{j}\right) e^{-\lambda_{j} V}}{\lambda_{j}^{2}\left(\lambda_{i}-\lambda_{j}\right)^{2}\left(\lambda_{j}-\lambda\right)}\right. \\
& \left.-\frac{2\left(1+V \lambda_{i}\right) e^{-\lambda_{i} V}}{\lambda_{i}^{2}\left(\lambda_{i}-\lambda_{j}\right)^{2}\left(\lambda_{i}-\lambda\right)}-\frac{\left(3 \lambda_{i}-\lambda_{j}-2 \lambda\right) e^{-\lambda_{i} V}}{\lambda_{i}\left(\lambda_{i}-\lambda_{j}\right)^{3}\left(\lambda_{i}-\lambda\right)^{3}}\right), \\
& \bar{M}_{X_{i} X_{j}}(V ; \lambda)=-2\left(\frac{e^{-\lambda V}}{\lambda\left(2 \lambda_{i}-\lambda\right)^{2}\left(\lambda_{j}-\lambda\right)^{2}}-\frac{\left(2 \lambda_{i}-3 \lambda_{j}+2 \lambda\right) e^{-\lambda_{j} V}}{\lambda_{j}\left(2 \lambda_{i}-\lambda_{j}\right)^{3}\left(\lambda_{j}-\lambda\right)^{2}}\right. \\
& -\frac{\left(1+V \lambda_{j}\right) e^{-\lambda_{j} V}}{\lambda_{j}^{2}\left(2 \lambda_{i}-\lambda_{j}\right)^{2}\left(\lambda_{j}-\lambda\right)}-\frac{2\left(1+V 2 \lambda_{i}\right) e^{-2 \lambda_{i} V}}{\left(2 \lambda_{i}\right)^{2}\left(2 \lambda_{i}-\lambda_{j}\right)^{2}\left(2 \lambda_{i}-\lambda\right)}-\frac{\left(6 \lambda_{i}-\lambda_{j}-2 \lambda\right) e^{-2 \lambda_{i} V}}{\left.2 \lambda_{i}\left(2 \lambda_{i}-\lambda_{j}\right)^{3}\left(2 \lambda_{i}-\lambda\right)^{3}\right),} \\
& \bar{N}_{X_{i} X_{j}}(V ; \lambda)=-2\left(\frac{\lambda^{2}}{\lambda\left(\lambda_{i}-\lambda\right)^{2}\left(2 \lambda_{j}-\lambda\right)^{2}}-\frac{\left(\lambda_{i}-6 \lambda_{j}+2 \lambda\right) e^{-2 \lambda_{j} V}}{2 \lambda_{j}\left(\lambda_{i}-2 \lambda_{j}\right)^{3}\left(2 \lambda_{j}-\lambda\right)^{2}}\right. \\
& -\frac{\left(1+V 2 \lambda_{j}\right) e^{-2 \lambda_{j} V}}{\left(2 \lambda_{j}\right)^{2}\left(\lambda_{i}-2 \lambda_{j}\right)^{2}\left(2 \lambda_{j}-\lambda\right)}-\frac{2\left(1+V \lambda_{i}\right) e^{-\lambda_{i} V}}{\lambda_{i}^{2}\left(\lambda_{i}-2 \lambda_{j}\right)^{2}\left(\lambda_{i}-\lambda\right)}-\frac{\left(3 \lambda_{i}-2 \lambda_{j}-2 \lambda\right) e^{-\lambda_{i} V}}{\left.\lambda_{i}\left(\lambda_{i}-2 \lambda_{j}\right)^{3}\left(\lambda_{i}-\lambda\right)^{3}\right),} \\
& \bar{O}_{X_{i} X_{j}}(V ; \lambda)=4\left(\frac{\left(3 \lambda_{i}-\lambda_{j}-\lambda\right) e^{-2 \lambda_{i} V}}{\lambda_{i}\left(2 \lambda_{i}-2 \lambda_{j}\right)^{3}\left(2 \lambda_{i}-\lambda\right)^{3}}+\frac{\left(\lambda_{i}-3 \lambda_{j}+\lambda\right) e^{-2 \lambda_{j} V}}{\lambda_{j}\left(2 \lambda_{i}-2 \lambda_{j}\right)^{3}\left(2 \lambda_{j}-\lambda\right)^{2}}\right. \\
& \left.+\frac{\left(1+V 2 \lambda_{j}\right) e^{-2 \lambda_{j} V}}{\left(2 \lambda_{j}\right)^{2}\left(2 \lambda_{i}-2 \lambda_{j}\right)^{2}\left(2 \lambda_{j}-\lambda\right)}+\frac{2\left(1+V 2 \lambda_{i}\right) e^{-2 \lambda_{i} V}}{\left(2 \lambda_{i}\right)^{2}\left(2 \lambda_{i}-2 \lambda_{j}\right)^{2}\left(2 \lambda_{i}-\lambda\right)}-\frac{e^{-\lambda V}}{\lambda\left(2 \lambda_{i}-\lambda\right)^{2}\left(2 \lambda_{j}-\lambda\right)^{2}}\right) .
\end{aligned}
$$


Now, we give the formulae of $E^{X_{i}^{3}}, i=1,2, \cdots, n$.

$$
E^{X_{i}^{3}}=\mathbf{E}\left[X_{i}^{3} \mid S>\operatorname{VaR}_{\kappa}(S)\right]=\frac{\int_{\operatorname{VaR}_{\kappa}(S)}^{\infty} \int_{0}^{s} x_{i}^{3} f_{X_{i}, S}\left(x_{i}, s\right) \mathrm{d} x_{i} \mathrm{~d} s}{1-\kappa} .
$$

Now, we rewrite the $f_{X_{1}, X_{2}, \cdots, X_{n}}\left(x_{1}, x_{2}, \cdots, x_{n}\right)$ defined in Eq.(2.2) as

$$
\begin{aligned}
& f_{X_{1}, X_{2}, \cdots, X_{n}}\left(x_{1}, x_{2}, \cdots, x_{n}\right)=f_{X_{i}}\left(x_{i}\right)\left\{\omega_{-i}\left(x_{1} ; x_{2} ; \cdots ; x_{n} ; \lambda_{1} ; \lambda_{2} ; \cdots ; \lambda_{n}\right)\right. \\
& +\sum_{q=2}^{n} \sum_{1 \leq p_{1}<\cdots<p_{q} \leq n \bigcap} \theta_{p_{1} \cdots p_{q}}\left(1-2 F_{X_{i}}\left(x_{i}\right)\right)\left[\sum_{l=0}^{q} \sum_{\left.\left(\epsilon_{1}, \cdots, \epsilon_{q}\right\}\right)-i \in \tau_{l, q-1}}\right. \\
& \left.(-1)^{l} \omega_{-i}\left(x_{p_{1}} ; x_{p_{2}} ; \cdots ; x_{p_{q}} ; x_{i_{q+1}} ; \cdots ; x_{i_{n}} ; 2^{\epsilon_{1}} \lambda_{p_{1}} ; \cdots ; 2^{\epsilon q} \lambda_{p_{q}} ; \lambda_{i_{q+1}} \cdots ; \lambda_{i_{n}}\right)\right] \\
& +\sum_{q=2}^{n} \sum_{1 \leq p_{1}<\cdots<p_{q} \leq n \bigcap i \notin\left\{p_{1}, \cdots, p_{q}\right\}} \theta_{p_{1} \cdots p_{q}}\left[\sum_{l=0}^{q} \sum_{\left(\epsilon_{1}, \cdots, \epsilon_{q}\right) \in \tau_{l, q}}\right. \\
& \left.(-1)^{l} \omega_{-i}\left(x_{p_{1}} ; x_{p_{2}} ; \cdots ; x_{p_{q}} ; x_{i_{q+1}} ; \cdots ; x_{i_{n}} ; 2^{\epsilon_{1}} \lambda_{p_{1}} ; \cdots ; 2^{\epsilon_{q}} \lambda_{p_{q}} ; \lambda_{i_{q+1}} \cdots ; \lambda_{i_{n}}\right)\right] \text {, }
\end{aligned}
$$

where $\omega_{-i}\left(x_{1} ; x_{2} ; \cdots ; x_{n} ; \alpha_{1} ; \alpha_{2} ; \cdots ; \alpha_{n}\right)=\alpha_{1} e^{-\alpha_{1} x_{1}} \times \cdots \times \alpha_{i-1} e^{-\alpha_{i-1} x_{i-1}} \times \alpha_{i+1} \times$ $e^{-\alpha_{i+1} x_{i+1}} \times \cdots \times \alpha_{n} e^{-\alpha_{n} x_{n}}$.

Assume that

$$
\begin{aligned}
& \int_{0}^{s-x_{i}} \int_{0}^{s-x_{1}-x_{i}} \ldots \int_{0}^{s-x_{1}-\cdots-x_{n-1}} \omega_{-i}\left(x_{1} ; \ldots ; x_{n} ; \alpha_{1} ; \ldots ; \alpha_{n}\right) \mathrm{d} x_{1} \cdots \mathrm{d} x_{i-1} \times \\
= & \sum_{l=1, l \neq i}^{n}\left(\prod_{q=1, q \neq l, q \neq i} \frac{\alpha_{q}}{\alpha_{q}-\alpha_{l}}\right) \alpha_{l} e^{-\alpha_{l}\left(s-x_{i}\right)}=h_{-i}\left(s-x_{i} ; \alpha_{1} ; \alpha_{2} ; \cdots ; \alpha_{n}\right) .
\end{aligned}
$$

Utilizing the dividend difference presented in Chiragiev and Landsman [6], note that

$$
\begin{gathered}
h_{-i}\left(x ; \alpha_{1} ; \cdots ; \alpha_{i-1} ; \alpha_{i+1} ; \cdots ; \alpha_{n}\right) \\
=(-1)^{n-2} \times \alpha_{1} \times \cdots \times \alpha_{i-1} \times \alpha_{i+1} \times \cdots \times \alpha_{n} \times \bar{\Lambda}\left(x ; \alpha_{1} ; \cdots ; \alpha_{i-1} ; \alpha_{i+1} ; \cdots ; \alpha_{n}\right),
\end{gathered}
$$

where $\bar{\Lambda}\left(x ; \alpha_{1} ; \cdots ; \alpha_{i-1} ; \alpha_{i+1} ; \cdots ; \alpha_{n}\right)$ is the $(n-2)$ th order divided difference of $\bar{\Lambda}(x ; \alpha)$. Then, we get

$$
\begin{gathered}
\int_{0}^{s} x_{i}^{3} f_{X_{i}}\left(x_{i}\right) h_{-i}\left(s-x_{i} ; \lambda_{1} ; \cdots ; \lambda_{i-1} ; \lambda_{i+1} ; \cdots ; \lambda_{n}\right) \mathrm{d} x_{i} \\
=(-1)^{n-1} \eta L_{X_{i}^{3}}\left(s ; \lambda_{1} ; \cdots ; \lambda_{i-1} ; \lambda_{i+1} ; \cdots ; \lambda_{j-1} ; \lambda_{j+1} ; \cdots ; \lambda_{n}\right),
\end{gathered}
$$

where $\eta_{-i}=\lambda_{1} \times \cdots \times \lambda_{i-1} \times \lambda_{i+1} \times \cdots \times \lambda_{n}, L_{X_{i}^{3}}(s ; \lambda)=-\int_{0}^{s} x_{i}^{3} e^{-\lambda_{i} x_{i}} \bar{\Lambda}\left(s-x_{i} ; \lambda\right) \mathrm{d} x_{i}$. Let also $M_{X_{i}^{3}}(s ; \lambda)=-\int_{0}^{s} 2 x_{i}^{3} e^{-2 \lambda_{i} x_{i}} \bar{\Lambda}\left(s-x_{i} ; \lambda\right) \mathrm{d} x_{i}$. Given that the risks are exponentially distributed, then $L_{X_{i}^{3}}$ and $M_{X_{i}^{3}}$ can be rewritten as

$$
\begin{gathered}
L_{X_{i}^{3}}(s ; \lambda)=\frac{s^{3} e^{-s \lambda_{i}}}{\lambda_{i}-\lambda}+\frac{3 s^{2} e^{-s \lambda_{i}}}{\left(\lambda_{i}-\lambda\right)^{2}}+\frac{6 s e^{-s \lambda_{i}}}{\left(\lambda_{i}-\lambda\right)^{3}}+\frac{6 e^{-s \lambda_{i}}}{\left(\lambda_{i}-\lambda\right)^{4}}-\frac{6 e^{-s \lambda}}{\left(\lambda_{i}-\lambda\right)^{4}}, \\
M_{X_{i}^{3}}(s ; \lambda)=2\left(\frac{s^{3} e^{-2 s \lambda_{i}}}{2 \lambda_{i}-\lambda}+\frac{3 s^{2} e^{-s \lambda_{i}}}{\left(\lambda_{i}-\lambda\right)^{2}}+\frac{6 s e^{-2 s \lambda_{i}}}{\left(2 \lambda_{i}-\lambda\right)^{3}}+\frac{6 e^{-s 2 \lambda_{i}}}{\left(2 \lambda_{i}-\lambda\right)^{4}}-\frac{6 e^{-s \lambda}}{\left(2 \lambda_{i}-\lambda\right)^{4}}\right) .
\end{gathered}
$$

Then, utilizing the results of (4.23), (4.25) and (4.26), we can express $\int_{0}^{s} x_{i}^{3} f_{X_{i}, S}\left(x_{i}, s\right) \mathrm{d} x_{i}$ as

$$
\int_{0}^{s} x^{3} f_{X_{i}, S}(x, s) \mathrm{d} x=(-1)^{n-1} \eta\left\{L_{X_{i}^{3}}\left(s ; \lambda_{1} ; \lambda_{2} ; \cdots ; \lambda_{n}\right)+\sum_{q=2}^{n} \sum_{1 \leq p_{1}<\cdots<p_{q} \leq n \bigcap i \in\left\{p_{1}, \cdots, p_{q}\right\}}\right.
$$




$$
\begin{gathered}
\theta_{p_{1} \cdots p_{q}}\left[\sum _ { l = 0 } ^ { q - 1 } \sum _ { ( \epsilon _ { 1 } , \cdots , \epsilon _ { q } ) - i \in \tau _ { l , q - 1 } } \left\{(-1)^{l+1} 2^{q-1-l} L_{X_{i}^{3}}\left(s ; 2^{\epsilon_{1}} \lambda_{p_{1}} ; \cdots ; 2^{\epsilon q} \lambda_{p_{q}} ; \lambda_{i_{q+1}} \cdots ; \lambda_{i_{n}}\right)\right.\right. \\
\left.\left.+(-1)^{l} 2^{q-1-l} M_{X_{i}^{3}}\left(s ; 2^{\epsilon_{1}} \lambda_{p_{1}} ; \cdots ; 2^{\epsilon_{q}} \lambda_{p_{q}} ; \lambda_{i_{q+1}} \cdots ; \lambda_{i_{n}}\right)\right\}\right]+\sum_{q=2}^{n} \sum_{1 \leq p_{1}<\cdots<p_{q} \leq n \bigcap i \notin\left\{p_{1}, \cdots, p_{q}\right\}} \\
\left.\theta_{p_{1} \cdots p_{q}} \times\left[\sum_{l=0}^{q} \sum_{\left(\epsilon_{1}, \cdots, \epsilon_{q}\right) \in \tau_{l, q}}(-1)^{l} 2^{q-l} L_{X_{i}^{3}}\left(s ; 2^{\epsilon_{1}} \lambda_{p_{1}} ; \cdots ; 2^{\epsilon_{q}} \lambda_{p_{q}} ; \lambda_{i_{q+1}} \cdots ; \lambda_{i_{n}}\right)\right]\right\} .
\end{gathered}
$$

In order to calculate the explicit the formulae of $E^{X_{i}^{3}}, i=1,2, \cdots, n$, as in (4.22), the $L_{X_{i}^{3}}(s ; \lambda)$ and $M_{X_{i}^{3}}(s ; \lambda)$ terms in the equation above must be integrated on $s$ as follows.

$$
\begin{gathered}
\bar{L}_{X_{i}^{3}}(V ; \lambda)=\int_{V}^{\infty} L_{X_{i}^{3}}(s ; \lambda) \mathrm{d} s=\frac{e^{-\lambda_{i} V}\left(6+V \lambda_{i}\left(6+V \lambda_{i}\left(3+V \lambda_{i}\right)\right)\right)}{\lambda_{i}^{4}\left(\lambda_{i}-\lambda\right)}+\frac{6 e^{-V \lambda_{i}}}{\lambda_{i}\left(\lambda_{i}-\lambda\right)^{4}} \\
-\frac{6 e^{-V \lambda}}{\lambda\left(\lambda_{i}-\lambda\right)^{4}}+\frac{3 e^{-V \lambda_{i}}\left(2+V \lambda_{i}\left(2+V \lambda_{i}\right)\right)}{\lambda_{i}^{3}\left(\lambda_{i}-\lambda\right)^{2}}+\frac{6 e^{-V \lambda_{i}}\left(1+V \lambda_{i}\right) e^{-V \lambda_{i}}}{\lambda_{i}^{2}\left(\lambda_{i}-\lambda\right)^{3}} . \\
\bar{M}_{X_{i}^{3}}(V ; \lambda)=\int_{V}^{\infty} M_{X_{i}^{3}}(s ; \lambda) \mathrm{d} s=\frac{e^{-2 \lambda_{i} V}\left(3+V \lambda_{i}\left(6+V 2 \lambda_{i}\left(3+V 2 \lambda_{i}\right)\right)\right)}{2^{2}\left(\lambda_{i}\right)^{4}\left(2 \lambda_{i}-\lambda\right)}-\frac{12 e^{-V \lambda}}{\lambda\left(2 \lambda_{i}-\lambda\right)^{4}} \\
+\frac{3 e^{-V 2 \lambda_{i}}\left(1+V \lambda_{i}\left(2+V 2 \lambda_{i}\right)\right)}{2\left(\lambda_{i}\right)^{3}\left(2 \lambda_{i}-\lambda\right)^{2}}+\frac{3 e^{-V 2 \lambda_{i}}\left(1+V 2 \lambda_{i}\right) e^{-V 2 \lambda_{i}}}{\left(\lambda_{i}\right)^{2}\left(2 \lambda_{i}-\lambda\right)^{3}}+\frac{6 e^{-V 2 \lambda_{i}}}{\lambda_{i}\left(2 \lambda_{i}-\lambda\right)^{4}} .
\end{gathered}
$$

Finally, we can derive the explicit formulae (4.3) for $E^{X_{i}^{3}}, i=1,2, \cdots, n$.

Since the formulae of $E^{X_{i}^{2}}$ and $E^{X_{i}}$ can be calculated similarly, we also omit the proof. We give the expressions of $\bar{L}_{X_{i}^{2}}(V ; \lambda), \bar{M}_{X_{i}^{2}}(V ; \lambda), \bar{L}_{X_{i}}(V ; \lambda)$ and $\bar{M}_{X_{i}}(V ; \lambda)$ directly,

$$
\begin{aligned}
& \bar{L}_{X_{i}^{2}}(V ; \lambda)=\frac{e^{-V \lambda_{i}}\left(2+V \lambda_{i}\left(2+V \lambda_{i}\right)\right)}{\lambda_{i}^{3}\left(\lambda_{i}-\lambda\right)}+\frac{2 e^{-V \lambda_{i}}\left(1+V \lambda_{i}\right) e^{-V \lambda_{i}}}{\lambda_{i}^{2}\left(\lambda_{i}-\lambda\right)^{2}} \\
& +\frac{2 e^{-V \lambda_{i}}}{\lambda_{i}\left(\lambda_{i}-\lambda\right)^{3}}-\frac{2 e^{-V \lambda}}{\lambda\left(\lambda_{i}-\lambda\right)^{3}} \\
& \bar{M}_{X_{i}^{2}}(V ; \lambda)=\frac{e^{-V 2 \lambda_{i}}\left(1+V \lambda_{i}\left(2+V 2 \lambda_{i}\right)\right)}{2^{2}\left(\lambda_{i}\right)^{3}\left(2 \lambda_{i}-\lambda\right)}+\frac{e^{-V 2 \lambda_{i}}\left(1+V 2 \lambda_{i}\right) e^{-V 2 \lambda_{i}}}{2\left(\lambda_{i}\right)^{2}\left(2 \lambda_{i}-\lambda\right)^{2}} \\
& +\frac{e^{-V 2 \lambda_{i}}}{\lambda_{i}\left(2 \lambda_{i}-\lambda\right)^{3}}-\frac{2 e^{-V \lambda}}{\lambda\left(2 \lambda_{i}-\lambda\right)^{3}} \\
& \bar{L}_{X_{i}}(V ; \lambda)=\frac{e^{-V \lambda_{i}}\left(1+V \lambda_{i}\right) e^{-V \lambda_{i}}}{\lambda_{i}^{2}\left(\lambda_{i}-\lambda\right)}+\frac{e^{-V \lambda_{i}}}{\lambda_{i}\left(\lambda_{i}-\lambda\right)^{2}}-\frac{e^{-V \lambda}}{\lambda\left(\lambda_{i}-\lambda\right)^{2}}, \\
& \bar{M}_{X_{i}}(V ; \lambda)=\frac{e^{-V 2 \lambda_{i}}\left(1+V 2 \lambda_{i}\right) e^{-V 2 \lambda_{i}}}{\left(2 \lambda_{i}\right)^{2}\left(2 \lambda_{i}-\lambda\right)}+\frac{e^{-V 2 \lambda_{i}}}{2 \lambda_{i}\left(2 \lambda_{i}-\lambda\right)^{2}}-\frac{e^{-V \lambda}}{\lambda\left(2 \lambda_{i}-\lambda\right)^{2}} .
\end{aligned}
$$

This completes the proof of this proposition.

\section{Numerical illustrations}

\subsection{Bivariate case}

We illustrate our results with an example for the bivariate case. As an example, assume that risk variables $X_{1}$ and $X_{2}$ are exponentially distributed with parameters $\lambda_{1}=2 / 5$ and $\lambda_{2}=3 / 4$. We calculate the optimal capital allocations based on Tail Mean-Variance principle for different risk levels $\kappa$, FGM copula parameters $\theta$ and different $\beta$.

The values of $d_{i}(i=1,2)$ with a total capital $d=40$ for $\theta=-1,0,1, \beta=0.1,0.3,0.5$, $0.7,0.9$, and $\kappa=0.5,0.75,0.95,0.99,0.995$ are listed in Table $1-3$. We observe that for 
fixed $\kappa$ and $\theta, d_{1}$ is a decreasing function with respect to $\beta$, but $d_{2}$ is an increasing function with respect to $\beta$. We also find that for fixed $\beta$ and $\theta, d_{1}$ is an increasing function with respect to $\kappa$, but $d_{2}$ is a decreasing function with respect to $\kappa$. Moreover, the larger the value of $\theta$, the greater changes in $d_{1}$ and $d_{2}$ for different $\kappa$ and $\beta$.

\subsection{Multivariate case}

For multivariate case, we illustrate our results with an example for three risk variables dependent through a trivariate FGM copula. Assume that risk variables $X_{1}, X_{2}$ and $X_{3}$ are exponentially distributed with parameters $\lambda_{1}=1 / 2, \lambda_{2}=1 / 3$ and $\lambda_{3}=1 / 5$. The trivariate FGM copula with 4 parameters can be written as

$$
C\left(u_{1}, u_{2}, u_{3}\right)=u_{1} u_{2} u_{3}\left(1+\theta_{123} \bar{u}_{1} \bar{u}_{2} \bar{u}_{3}+\theta_{12} \bar{u}_{1} \bar{u}_{2}+\theta_{13} \bar{u}_{1} \bar{u}_{3}+\theta_{23} \bar{u}_{2} \bar{u}_{3}\right),
$$

where $\bar{u}_{i}=1-u_{i}, i=1,2,3$.

We calculate the optimal capital allocations (the amount $d_{i}$ to $X_{i}, i=1,2,3$ ) based on Tail Mean-Variance principle for different risk levels $\kappa$, FGM copula parameters and different $\beta$.

The values of $d_{i}(i=1,2,3)$ with a total capital $d=120$ for $\beta=0.1,0.3,0.5,0.7,0.9$, $\kappa=0.5,0.75,0.95,0.99,0.995, \theta_{123}=0.25, \theta_{12}=-0.2,0.2, \theta_{13}=0.5$, and $\theta_{23}=-0.6,0.6$ are listed in Table 4-5. We observe that for fixed $\kappa, d_{2}$ and $d_{3}$ are decreasing functions with respect to $\beta$, but $d_{1}$ is an increasing function with respect to $\beta$ in both cases. We also find that for fixed $\beta, d_{3}$ is an increasing function with respect to $\kappa$, but $d_{1}$ and $d_{2}$ is decreasing function with respect to $\kappa$. Moreover, the changes in $d_{1}, d_{2}$ and $d_{2}$ for different $\kappa$ and $\beta$ in case $\theta_{123}=0.25, \theta_{12}=0.2, \theta_{13}=0.5$ and $\theta_{23}=0.6$ are greater than in case $\theta_{123}=0.25, \theta_{12}=-0.2, \theta_{13}=0.5$ and $\theta_{23}=-0.6$.

\section{Concluding Remarks}

In this paper, we introduce the use of copulas in optimal capital allocation based on Tail Mean-Variance principle. We obtain explicit expressions for the optimal capital allocation for exponential distributed risks linked by a FGM copula. The handy form of the FGM copula permits a direct calculation of the $\mathbf{E}\left[X_{i}^{k} \mid S>\operatorname{VaR}_{\kappa}(S)\right], \mathbf{E}\left[X_{i}^{2} X_{j} \mid S>\right.$ $\left.\operatorname{VaR}_{\kappa}(S)\right]$, and $\mathbf{E}\left[X_{i} X_{j} \mid S>\operatorname{VaR}_{\kappa}(S)\right]$ when we assume only two risks. In the multivariate case, the dividend differences presented in Chiragiev and Landsman (2007) are used.

\section{Acknowledgements}

The authors thank the Editor and two anonymous reviewers for their comments that helped to improve the presentation of this paper. Xie's research was supported by the Natural Science Foundation of China (Grant No. 11561047), the Social Science Foundation of JiangXi Province (Grant No. 16YJ28), and the STRP of JiangXi Province (Grant No. GJJ151116). Zou's research was supported by the STRP of JiangXi Province (Grant No.GJJ151101).

\section{References}

[1] Bargès, M., Cossette, H. and Marceau, E. TVaR-based capital allocation with copulas, Insurance: Mathematics and Economics, 45, 348-361, 2009.

[2] Bauer, D. and Zanjani, G. Capital allocation and its discontents, In Handbook of Insurance (pp 863-880), Springer New York, 2013.

[3] Bauer, D. and Zanjani, G. The marginal cost of risk, risk measures, and capital allocation, Management Science 62, 1431-1457, 2015. 
[4] Belles-Sampera, J., Guillén, M. and Santolino, M. GlueVaR risk measures in capital allocation applications, Insurance: Mathematics and Economics 58, 132137,2014

[5] Cai, J. and Wei, W. Some new notions of dependence with applications in optimal allocation problems, Insurance: Mathematics and Economics 55, 200-209, 2014.

[6] Chiragiev. A and Landsman, Z. Multivariate pareto portfolios: Tce-based capital allocation and divided differences, Scandinavian Actuarial Journal 4, 261-280, 2007.

[7] Cossette, H., Côté, M., Marceau, E. and Moutanabbir, K. Multivariate distribution defined with Farlie-Gumbel-Morgenstern copula and mixed Erlang marginals: Aggregation and capital allocation, Insurance: Mathematics and Economics 52, 560-572, 2013.

[8] Cossette, H., Marceau, E. and Marri, F. On a compound Poisson risk model with dependence and in the presence of a constant dividend barrier, Applied Stochastic Models in Business and Industry 30, 82-98, 2014.

[9] Dhaene, J., Tsanakas, A., Valdez, E. and Vanduffel, S. Optimal capital allocation principles, Journal of Risk and Insurance 79, 1-28, 2012.

[10] Frostig, E., Zaks, Y. and Levikson, B. Optimal pricing for a heterogeneous portfolio for a given risk factor and convex distance measure, Insurance: Mathematics and Economics 40, 459-467, 2007.

[11] Gebizlioglu, O. and Yagci, B. Tolerance intervals for quantities of bivariate risks and risk measurement. Insurance: Mathematics and Economics 42, 1022-1027, 2008.

[12] Geluk, J. and Tang, Q. Asymptotic tail probabilities of sums of dependent subexponential random variables. Journal of Theoretical Probability 22, 871-882, 2009.

[13] Laeven, R.J.A. and Goovaerts, M.J. An optimization approach to the dynamic allocation of economic capital, Insurance: Mathematics and Economics 35, 299$319,2004$.

[14] Manesh, S.F. and Khaledi, B.E. Allocations of policy limits and ordering relations for aggregate remaining claims, Insurance: Mathematics and Economics 65, 9-14, 2015.

[15] Nelsen, R. B. An introduction to copulas (2nd ed), Springer Series in Statistics, New York: Springer-Verlag, 2006.

[16] Xie, J.H. and Zou, W. On the expected discounted penalty function for a risk model with dependence under a multi-layer dividend strategy, Communications in Statistics-Theory and Methods 46, 1898-1915, 2017.

[17] Xu, M. and Hu, T. Stochastic comparisons of capital allocations with applications. Insurance: Mathematics and Economics 50, 293-298, 2012.

[18] Xu, M. and Mao, T. Optimal capital allocation based on the Tail Mean-Variance Model, Insurance: Mathematics and Economics 53, 533-543, 2013.

[19] You, Y.P. and Li, X.H. Optimal capital allocations to interdependent actuarial risks, Insurance: Mathematics and Economics, 57, 104-113, 2014.

[20] Zaks, Y., Frosting, E. and Levikson, B. Optimal pricing of a heterogeneous portfolio for a given risk level, Astin Bulletin 36, 161-185, 2006.

[21] Zaks, Y. and Tsanakas, A. Optimal capital allocation in a hierarchical corporate structure, Insurance Mathematics and Economics 56, 48-55, 2014. 
Table 1. Optimal capital allocations for different $\beta$ and $\kappa$ with a total capital $d=40$ and $\theta=-1$.

\begin{tabular}{|c|c|c|c|c|c|}
\hline$\beta$ & $\kappa=0.5$ & $\kappa=0.75$ & $\kappa=0.95$ & $\kappa=0.99$ & $\kappa=0.995$ \\
\hline$\beta=0.1$ & $d_{1}=17.373255$ & $d_{1}=18.259265$ & $d_{1}=19.786721$ & $d_{1}=21.285369$ & $d_{1}=22.077165$ \\
& $d_{2}=22.626745$ & $d_{2}=21.740735$ & $d_{2}=20.213279$ & $d_{2}=18.714631$ & $d_{2}=17.922835$ \\
\hline$\beta=0.3$ & $d_{1}=16.657079$ & $d_{1}=17.760345$ & $d_{1}=19.374409$ & $d_{1}=20.740284$ & $d_{1}=21.438334$ \\
& $d_{2}=23.342921$ & $d_{2}=22.239655$ & $d_{2}=20.625591$ & $d_{2}=19.259716$ & $d_{2}=18.561666$ \\
\hline$\beta=0.5$ & $d_{1}=16.479421$ & $d_{1}=17.642965$ & $d_{1}=19.281445$ & $d_{1}=20.615971$ & $d_{1}=21.290319$ \\
& $d_{2}=23.520579$ & $d_{2}=22.357035$ & $d_{2}=20.718555$ & $d_{2}=19.384029$ & $d_{2}=18.709681$ \\
\hline$\beta=0.7$ & $d_{1}=16.398849$ & $d_{1}=17.590521$ & $d_{1}=19.240384$ & $d_{1}=20.560895$ & $d_{1}=21.224463$ \\
& $d_{2}=23.601151$ & $d_{2}=22.409479$ & $d_{2}=20.759616$ & $d_{2}=19.439105$ & $d_{2}=18.775537$ \\
\hline$\beta=0.9$ & $d_{1}=16.352859$ & $d_{1}=17.560802$ & $d_{1}=19.217245$ & $d_{1}=20.529814$ & $d_{1}=21.187221$ \\
& $d_{2}=23.647141$ & $d_{2}=22.439198$ & $d_{2}=20.782755$ & $d_{2}=19.470186$ & $d_{2}=18.812779$ \\
\hline
\end{tabular}

Table 2. Optimal capital allocations for different $\beta$ and $\kappa$ with a total capital $d=40$ and $\theta=0$.

\begin{tabular}{|c|c|c|c|c|c|}
\hline$\beta$ & $\kappa=0.5$ & $\kappa=0.75$ & $\kappa=0.95$ & $\kappa=0.99$ & $\kappa=0.995$ \\
\hline$\beta=0.1$ & $d_{1}=17.085053$ & $d_{1}=18.257321$ & $d_{1}=20.322754$ & $d_{1}=22.219689$ & $d_{1}=23.064009$ \\
& $d_{2}=22.914947$ & $d_{2}=21.742679$ & $d_{2}=19.677246$ & $d_{2}=17.780311$ & $d_{2}=16.935991$ \\
\hline$\beta=0.3$ & $d_{1}=16.182965$ & $d_{1}=17.688514$ & $d_{1}=19.991725$ & $d_{1}=21.946054$ & $d_{1}=22.801869$ \\
& $d_{2}=23.817035$ & $d_{2}=22.311486$ & $d_{2}=20.008275$ & $d_{2}=18.053946$ & $d_{2}=17.198131$ \\
\hline$\beta=0.5$ & $d_{1}=15.951057$ & $d_{1}=17.551042$ & $d_{1}=19.916478$ & $d_{1}=21.885122$ & $d_{1}=22.743742$ \\
& $d_{2}=24.048943$ & $d_{2}=22.448958$ & $d_{2}=20.083522$ & $d_{2}=18.114878$ & $d_{2}=17.256258$ \\
\hline$\beta=0.7$ & $d_{1}=15.844774$ & $d_{1}=17.489157$ & $d_{1}=19.883171$ & $d_{1}=21.858299$ & $d_{1}=22.718181$ \\
& $d_{2}=24.155226$ & $d_{2}=22.510843$ & $d_{2}=20.116829$ & $d_{2}=18.141701$ & $d_{2}=17.281819$ \\
\hline$\beta=0.9$ & $d_{1}=15.783795$ & $d_{1}=17.453962$ & $d_{1}=19.864381$ & $d_{1}=21.843206$ & $d_{1}=22.703806$ \\
& $d_{2}=24.216205$ & $d_{2}=22.546038$ & $d_{2}=20.135619$ & $d_{2}=18.156794$ & $d_{2}=17.296194$ \\
\hline
\end{tabular}

Table 3. Optimal capital allocations for different $\beta$ and $\kappa$ with a total capital $d=40$ and $\theta=1$.

\begin{tabular}{|c|c|c|c|c|c|}
\hline$\beta$ & $\kappa=0.5$ & $\kappa=0.75$ & $\kappa=0.95$ & $\kappa=0.99$ & $\kappa=0.995$ \\
\hline$\beta=0.1$ & $d_{1}=16.663625$ & $d_{1}=18.058854$ & $d_{1}=20.322699$ & $d_{1}=22.208001$ & $d_{1}=23.017989$ \\
& $d_{2}=23.336375$ & $d_{2}=21.941146$ & $d_{2}=19.677301$ & $d_{2}=17.791999$ & $d_{2}=16.982011$ \\
\hline$\beta=0.3$ & $d_{1}=15.445589$ & $d_{1}=17.357541$ & $d_{1}=19.991519$ & $d_{1}=21.965215$ & $d_{1}=22.791365$ \\
& $d_{2}=24.554411$ & $d_{2}=22.642459$ & $d_{2}=20.008481$ & $d_{2}=18.034785$ & $d_{2}=17.208635$ \\
\hline$\beta=0.5$ & $d_{1}=15.115614$ & $d_{1}=17.181922$ & $d_{1}=19.915242$ & $d_{1}=21.911007$ & $d_{1}=22.741114$ \\
& $d_{2}=24.884386$ & $d_{2}=22.818078$ & $d_{2}=20.084758$ & $d_{2}=18.088993$ & $d_{2}=17.258886$ \\
\hline$\beta=0.7$ & $d_{1}=14.961949$ & $d_{1}=17.102057$ & $d_{1}=19.881358$ & $d_{1}=21.887125$ & $d_{1}=22.719015$ \\
& $d_{2}=25.038051$ & $d_{2}=22.897943$ & $d_{2}=20.118642$ & $d_{2}=18.112875$ & $d_{2}=17.280985$ \\
\hline$\beta=0.9$ & $d_{1}=14.873078$ & $d_{1}=17.056409$ & $d_{1}=19.862212$ & $d_{1}=21.873684$ & $d_{1}=22.706589$ \\
& $d_{2}=25.126922$ & $d_{2}=22.943591$ & $d_{2}=20.137788$ & $d_{2}=18.126316$ & $d_{2}=17.293411$ \\
\hline
\end{tabular}


Table 4. Optimal capital allocations with $d=120$ and $\theta_{123}=0.25, \theta_{12}=-0.2$, $\theta_{13}=0.5, \theta_{23}=-0.6$.

\begin{tabular}{|c|c|c|c|c|c|}
\hline$\beta$ & $\kappa=0.5$ & $\kappa=0.75$ & $\kappa=0.95$ & $\kappa=0.99$ & $\kappa=0.995$ \\
\hline$\beta=0.1$ & $d_{1}=48.9773$ & $d_{1}=45.7090$ & $d_{1}=40.7692$ & $d_{1}=36.6939$ & $d_{1}=35.0049$ \\
& $d_{2}=40.7396$ & $d_{2}=40.2660$ & $d_{2}=39.5750$ & $d_{2}=38.9001$ & $d_{2}=38.4863$ \\
& $d_{3}=30.2831$ & $d_{3}=34.0250$ & $d_{3}=39.6558$ & $d_{3}=44.4060$ & $d_{3}=46.5088$ \\
\hline$\beta=0.3$ & $d_{1}=50.5392$ & $d_{1}=46.6406$ & $d_{1}=41.2002$ & $d_{1}=36.9332$ & $d_{1}=35.1863$ \\
& $d_{2}=40.1102$ & $d_{2}=39.9010$ & $d_{2}=39.4434$ & $d_{2}=38.8840$ & $d_{2}=38.5102$ \\
& $d_{3}=29.3506$ & $d_{3}=33.4584$ & $d_{3}=39.3564$ & $d_{3}=44.1828$ & $d_{3}=46.3035$ \\
\hline$\beta=0.5$ & $d_{1}=50.9125$ & $d_{1}=46.8548$ & $d_{1}=41.2958$ & $d_{1}=36.9856$ & $d_{1}=35.2258$ \\
& $d_{2}=39.9472$ & $d_{2}=39.8112$ & $d_{2}=39.4116$ & $d_{2}=38.8784$ & $d_{2}=38.5135$ \\
& $d_{3}=29.1403$ & $d_{3}=33.3340$ & $d_{3}=39.2926$ & $d_{3}=44.1360$ & $d_{3}=46.2607$ \\
\hline$\beta=0.7$ & $d_{1}=51.0801$ & $d_{1}=46.9500$ & $d_{1}=41.3378$ & $d_{1}=37.0085$ & $d_{1}=35.2431$ \\
& $d_{2}=39.8727$ & $d_{2}=39.7707$ & $d_{2}=39.3973$ & $d_{2}=38.8757$ & $d_{2}=38.5146$ \\
& $d_{3}=29.0472$ & $d_{3}=33.2793$ & $d_{3}=39.2649$ & $d_{3}=44.1158$ & $d_{3}=46.2423$ \\
\hline$\beta=0.9$ & $d_{1}=51.1753$ & $d_{1}=47.0038$ & $d_{1}=41.3614$ & $d_{1}=37.0213$ & $d_{1}=35.2527$ \\
& $d_{2}=39.8300$ & $d_{2}=39.7476$ & $d_{2}=39.3893$ & $d_{2}=38.8742$ & $d_{2}=38.5154$ \\
& $d_{3}=28.9947$ & $d_{3}=33.2486$ & $d_{3}=39.2493$ & $d_{3}=44.1045$ & $d_{3}=46.2319$ \\
\hline
\end{tabular}

Table 5. Optimal capital allocations with $d=120$ and $\theta_{123}=0.25, \theta_{12}=0.2$, $\theta_{13}=0.5, \theta_{23}=0.6$.

\begin{tabular}{|c|c|c|c|c|c|}
\hline$\beta$ & $\kappa=0.5$ & $\kappa=0.75$ & $\kappa=0.95$ & $\kappa=0.99$ & $\kappa=0.995$ \\
\hline$\beta=0.1$ & $d_{1}=53.9283$ & $d_{1}=49.0771$ & $d_{1}=42.6048$ & $d_{1}=38.0363$ & $d_{1}=36.2516$ \\
& $d_{2}=38.0905$ & $d_{2}=38.1029$ & $d_{2}=37.7804$ & $d_{2}=37.2270$ & $d_{2}=36.8990$ \\
& $d_{3}=27.9812$ & $d_{3}=32.8200$ & $d_{3}=39.6148$ & $d_{3}=44.7367$ & $d_{3}=46.8494$ \\
\hline$\beta=0.3$ & $d_{1}=56.0911$ & $d_{1}=50.4378$ & $d_{1}=43.3266$ & $d_{1}=38.5192$ & $d_{1}=36.6674$ \\
& $d_{2}=37.0629$ & $d_{2}=37.4505$ & $d_{2}=37.4379$ & $d_{2}=37.0155$ & $d_{2}=36.7271$ \\
& $d_{3}=26.8460$ & $d_{3}=32.1117$ & $d_{3}=39.2355$ & $d_{3}=44.4653$ & $d_{3}=46.6055$ \\
\hline$\beta=0.5$ & $d_{1}=56.6026$ & $d_{1}=50.7502$ & $d_{1}=43.4875$ & $d_{1}=38.6257$ & $d_{1}=36.7588$ \\
& $d_{2}=36.8042$ & $d_{2}=37.2938$ & $d_{2}=37.3591$ & $d_{2}=36.9671$ & $d_{2}=36.6877$ \\
& $d_{3}=26.5932$ & $d_{3}=31.9560$ & $d_{3}=39.1534$ & $d_{3}=44.4072$ & $d_{3}=46.5535$ \\
\hline$\beta=0.7$ & $d_{1}=56.8317$ & $d_{1}=50.8888$ & $d_{1}=43.5584$ & $d_{1}=38.6725$ & $d_{1}=36.7989$ \\
& $d_{2}=36.6865$ & $d_{2}=37.2235$ & $d_{2}=37.3241$ & $d_{2}=36.9457$ & $d_{2}=36.6703$ \\
& $d_{3}=26.4818$ & $d_{3}=31.8877$ & $d_{3}=39.1175$ & $d_{3}=44.3818$ & $d_{3}=46.5308$ \\
\hline$\beta=0.9$ & $d_{1}=56.9616$ & $d_{1}=50.9672$ & $d_{1}=43.5983$ & $d_{1}=38.6987$ & $d_{1}=36.8215$ \\
& $d_{2}=36.6193$ & $d_{2}=37.1835$ & $d_{2}=37.3043$ & $d_{2}=36.9337$ & $d_{2}=36.6604$ \\
& $d_{3}=26.4191$ & $d_{3}=31.8493$ & $d_{3}=39.0974$ & $d_{3}=44.3676$ & $d_{3}=46.5181$ \\
\hline
\end{tabular}

